\title{
EQUIVARIANT HIRZEBRUCH CLASSES AND MOLIEN SERIES OF QUOTIENT SINGULARITIES
}

\author{
MARIA DONTEN-BURY* \\ University of Warsaw \\ Institute of Mathematics \\ Banacha 2 \\ 02-097 Warszawa, Poland \\ and \\ Freie Universität Berlin \\ Institute of Mathematics \\ Arnimallee 3 \\ 14195 Berlin, Germany \\ m.donten@mimuw.edu.pl
}

\author{
ANDRZEJ WEBER \\ University of Warsaw \\ Institute of Mathematics \\ Banacha 2 \\ 02-097 Warszawa, Poland \\ and \\ Institute of Mathematics \\ Polish Academy of Sciences \\ ul. Śniadeckich 8 \\ 00-656 Warszawa, Poland \\ aweber@mimuw.edu.pl
}

\begin{abstract}
We study properties of the Hirzebruch class of quotient singularities $\mathbb{C}^{n} / G$, where $G$ is a finite matrix group. The main result states that the Hirzebruch class coincides with the Molien series of $G$ under suitable substitution of variables. The Hirzebruch class of a crepant resolution can be described specializing the orbifold elliptic genus constructed by Borisov and Libgober. It is equal to the combination of Molien series of centralizers of elements of $G$. This is an incarnation of the McKay correspondence. The results are illustrated with several examples, in particular of 4-dimensional symplectic quotient singularities.
\end{abstract}

\section{Introduction}

The McKay correspondence is a postulated relation between the geometry of a crepant resolution of a quotient singularity and the properties of the group defining the singularity, or its representation theory, see [IR96], [Rei97]. A resolution of singularities $\pi: Y \rightarrow X$ is called crepant if $\varphi^{*} K_{X}=K_{Y}$. One may think that this condition is to ensure that the resolution is, in some sense, "not too big", it does not have unnecessary components. It was first observed by McKay in the 2-dimensional case, $[\mathrm{McK} 80]$, that the structure of the minimal resolution of a Du Val singularity, $\mathbb{C}^{2} / G$ for $G \subset \mathrm{SL}(2, \mathbb{C})$, can be described in terms of the group structure: the components of the exceptional fiber correspond to conjugacy classes of elements of $G$, or to its irreducible representations. Reid

DOI: $10.1007 /$ s00031-017-9452-7

*Both authors supported by NCN grant 2013/08/A/ST1/00804. This work was completed while MD-B held a Dahlem Research School Postdoctoral Fellowship at Freie Universität Berlin.

Received July 6, 2016. Accepted January 24, 2017.

Published online November 13, 2017.

Corresponding Author: A. Weber, e-mail: aweber@mimuw.edu.pl 
conjectured, see [Rei97], that this should be true in a more general setting. Since then the correspondence was proven in certain cases on various levels of detail. First came the proof in dimension 3, given, e.g., in [IR96]. The weak version, i.e., the equality between $\operatorname{dim} H^{*}(E)$, where $E$ is the exceptional divisor, and the number of conjugacy classes in $G$ is due to Batyrev for any $G \subset \operatorname{SL}(n, \mathbb{C})$, [Bat99]. For symplectic resolutions of symplectic quotient singularities Kaledin has shown that there is a natural bijection between conjugacy classes in $G$ and the basis of cohomology, [Kal02]. The correspondence conjecture can be also rephrased in the language of derived categories, see [BKR01].

There is a broader understanding of the McKay correspondence. We search for a relation between the geometric properties of the resolution and the algebraic properties of the group and its representation. Here we do not assume that the resolution is crepant. It can be any resolution, but we pay a price: we have to correct the data if the resolution is too big. This is the strategy of [BL05] or [Vey03], also present in [Bat99]. The correction terms depend on the structure of the resolution. The invariant which we are interested in is the Hirzebruch class

$$
\operatorname{td}_{y}(X) \in H_{*}(X) \otimes \mathbb{Q}[y]
$$

It is defined both for the quotient variety, which is singular in general, and its resolution. If $X$ admits a crepant resolution $f: \widetilde{X} \rightarrow X$ then $\operatorname{td}_{y=0}(X)$ coincides with the image of the classical Todd class of the resolution

$$
\operatorname{td}_{y=0}(X)=f_{*} \operatorname{td}(\tilde{X}) .
$$

The equality does not hold for the full Hirzebruch class, as it is seen already by the example of Du Val singularities. However, the difference between $\operatorname{td}_{y}(X \hookrightarrow M)$ and $f_{*} \operatorname{td}_{y}(\tilde{X})$ is well controlled.

It is convenient to assume that $X$ is embedded in an ambient smooth variety $M$ and to study the image

$$
\operatorname{td}_{y}(X \hookrightarrow M) \in H_{*}(M) \otimes \mathbb{Q}[y] \simeq H^{*}(M) \otimes \mathbb{Q}[y]
$$

Since we are interested in equivariant situation with respect to the torus $\mathbb{C}^{*}$ action, our enriched invariant belongs to the equivariant cohomology

$$
\widehat{H}_{\mathbb{C}^{*}}^{*}(X) \otimes \mathbb{Q}[y]=\left(\prod_{k=0}^{\infty} H_{\mathbb{C}^{*}}^{k}(X)\right) \otimes \mathbb{Q}[y] .
$$

To compute the Hirzebruch class we use its functorial and motivic properties [BSY10, Thm. 3.1], see also [Web16b, §5]:

$$
\operatorname{td}_{y}(X \hookrightarrow M)=p_{*} \operatorname{td}_{y}(\tilde{X})-p_{*} \operatorname{td}_{y}(E \hookrightarrow \widetilde{X})+\operatorname{td}_{y}\left(p_{*}(E) \hookrightarrow M\right) .
$$

Here $p: \widetilde{X} \rightarrow X$ is the resolution of singularities and $E \subset \widetilde{X}$ is the exceptional divisor. The formula (1) can be treated as an inductive definition of the Hirzebruch class for singular varieties, provided that we know what $\operatorname{td}_{y}(X)$ is for a smooth 
variety as the initial step of the induction. In the smooth case the $\operatorname{class} \operatorname{td}_{y}(X)=$ $\operatorname{td}(T X) \operatorname{ch}\left(\Lambda_{y}\left(T^{*} X\right)\right)$ was defined by Hirzebruch [Hir56, Chap. 4]. (We recommend [Huy05, §5.1] as a short introduction.) It is the multiplicative characteristic class associated to the formal power series

$$
h_{y}(x)=x \frac{1+y e^{-x}}{1-e^{-x}}
$$

The Hirzebruch class for singular varieties was defined in [BSY10]. The equivariant version is studied in [Web16a], using the method of [Ohm06], see also the introductions to [MW15], [Web16b]. We wish to study singularities locally, therefore we restrict the Hirzebruch class to the singular point, or equivalently to any contractible $\mathbb{C}^{*}$-stable neighbourhood of the singular point. We will assume that the ambient space $M$ is a vector space with a linear action of $\mathbb{C}^{*}$. This way we obtain the local equivariant Hirzebruch class which belongs to the equivariant cohomology

$$
\widehat{H}_{\mathbb{C}^{*}}^{*}(M) \otimes \mathbb{Q}[y] \simeq \widehat{H}_{\mathbb{C}^{*}}^{*}(p t) \otimes \mathbb{Q}[y] \simeq \mathbb{Q}[[t]][y] .
$$

One can express the Hirzebruch class in a convenient way by taking $T=e^{-t}$ and using the Euler class of the $\mathbb{C}^{*}$-representation on the tangent space $T_{0} M \simeq M$. Our first result, Theorem 1, says that the Hirzebruch class of the quotient $M / G$ essentially coincides with the (extended) Molien series of the representation of $G$ on $M \simeq \mathbb{C}^{n}$.

A fundamental problem of the McKay correspondence is to compute invariants of a crepant resolution of a quotient singularity in terms of invariants of the action of $G$. In our case we consider quotients of an affine space, but to compute the Hirzebruch class of a crepant resolution (if it exists) we recall a construction which holds in general, and moreover is valid in the equivariant setup. The general theory is built in a series of papers of Borisov and Libgober [BL00], [BL03], [BL05]. They define a more delicate invariant, called the elliptic genus, and a related cohomology class. It is not motivic, i.e., it does not behave additively with respect to cut and paste operations. Nevertheless, the elliptic class can be defined for a certain class of singular varieties, including quotient singularities.

Due to [BL05] the elliptic class of $X / G$ can be computed in terms of various data associated to the fixed point sets $X^{g}$ for $g \in G$. If $X / G$ has a crepant resolution, then the elliptic class of $X / G$ is the image of the elliptic class of the resolution. If $X=V$ is an affine space we obtain a rather complicated description of the equivariant elliptic class of $V / G$ in purely algebraic terms. The elliptic class (depending on a formal parameter $q$ ) specializes to the Hirzebruch class when $q$ tends to 0 . This way, somehow going around, we arrive at the formula for the Hirzebruch class of a crepant resolution of $V / G$. This time the formula is easy. We obtain a combination of Molien series of centralizers of elements of $G$. The formula makes sense even when a crepant resolution does not exist.

The results presented here fit in the general idea of describing the geometry of resolutions of a quotient singularity in terms of group properties. In Theorem 1 we show that the equivariant Hirzebruch class of a quotient singularity $\mathbb{C}^{n} / G$ is very closely related to (and can be easily derived from) the (extended) Molien 
series of $G$, containing information about the degrees of invariants of exterior and symmetric powers of the considered representation of $G$. For us the symplectic actions are the most interesting. Their Hirzebruch class has a very particular form. We have computed multiple examples (mainly in dimension 4) and we have noticed certain phenomena, which deserve further study.

Maybe the most intriguing one is certain positivity of the local equivariant Hirzebruch class. The search for positivity of singular characteristic classes was started by Aluffi and Mihalcea in [AM09]. They observed that Chern-SchwartzMacPherson classes of Schubert cells in the classical Grassmannians are effective in all computed cases. Being effective (in that case) is equivalent to having nonnegative intersections with the basis of the cohomology of the Grassmannian formed by Schubert classes. It was conjectured that positivity holds for Grassmannians. The conjecture was proven by Huh [Huh16]. His proof works only for classical Grassmannians, while the question of positivity makes sense for any homogeneous space $G / P$. Also, the proof of Huh cannot be repeated in the equivariant setting.

Positivity of local equivariant Chern-Schwartz-MacPherson classes for Schubert varieties was noticed in [Web12] by computer experiments. So far there is no proof. Moreover, in [Web16a], it was noticed that there is another, stronger positivity of local equivariant Hirzebruch classes. Positivity was proven for simplicial toric varieties, while for various Schubert cells in $G / P$ it was only observed in the results of computations. The positivity of local equivariant Hirzebruch classes implies the positivity of local Chern-Schwartz-MacPherson classes. In the present paper we prove a particular form of positivity of local equivariant Hirzebruch classes as well as positivity of local equivariant Chern-Schwartz-MacPherson classes of quotient varieties $\mathbb{C}^{n} / G$.

We would like to remark that we do not know what are the meanings and consequences of both positivities. It is just a phenomenon, which we observe and prove in some cases. We wish to have applications and to find relations with other properties of singularities.

The results of the paper are the following:

- Theorem 1 shows that essentially the equivariant Hirzebruch class of $\mathbb{C}^{n} / G$ is equal to the Molien series of the representation. The proof is based on a version of the Lefschetz-Riemann-Roch theorem proved in [CMSS12]. It is given in Section 3.

- In Section 4 there are described symmetries of the Hirzebruch class of the quotient singularity. The proof is based on the interpretation of the equivariant Hirzebruch class as the Molien series. This is done in Section 4. For $G \subset \mathrm{SL}_{n}(\mathbb{C})$ or symplectic quotients we have additional functional equations.

- We illustrate Theorem 1 by the example of Du Val singularities in Section 5.

- Theorem 13 and Corollary 14 in Section 6 describe the equivariant Hirzebruch class of a crepant resolution in terms of Molien series. The result is a specialization of the McKay correspondence proved for the elliptic class by Borisov and Libgober [BL05]. 
- In Section 7 we discuss positivity of the local equivariant Hirzebruch class.

- In Appendix 8 we give a series of examples of Hirzebruch classes for symplectic quotients.

The symplectic singularities were in the center of our interest. Their crepant resolutions are automatically hyperkähler. For this class of singularities probably one should define and study a characteristic class which would contain more information than the elliptic class. We leave this subject for future research.

\section{The main result}

Suppose $G \subset \mathrm{GL}_{n}(\mathbb{C})$ is a finite group. In general the quotient $X=\mathbb{C}^{n} / G$ is a singular variety which admits an action of $\mathbb{C}^{*}$ coming from scalar multiplication in $\mathbb{C}^{n}$. Suppose $X$ is embedded equivariantly in a vector space $M$. As $M^{*}$ we can take the space freely spanned by the generators of $\left(\operatorname{Sym}^{\bullet}\left(\mathbb{C}^{n}\right)\right)^{G}$. Precisely, the set of homogeneous generators $s_{1}, s_{2}, \ldots, s_{m} \in\left(\operatorname{Sym}^{\bullet}\left(\mathbb{C}^{n}\right)\right)^{G}$ defines a surjection from the polynomial ring $\mathbb{C}\left[\tilde{s}_{1}, \tilde{s}_{2}, \ldots, \tilde{s}_{m}\right] \rightarrow\left(\operatorname{Sym} \bullet\left(\mathbb{C}^{n}\right)\right)^{G}$. This defines an embedding $\mathbb{C}^{n} / G \hookrightarrow M=\mathbb{C}^{m}$. The vector space $M$ admits a linear action of $\mathbb{C}^{*}$ such that the embedding is equivariant. The weights of this action are $w_{k}=\operatorname{deg}\left(s_{k}\right)$ for $k=1,2, \ldots, m$. The equivariant Hirzebruch class (see, e.g., [BSY10], [Web16a])

$$
\operatorname{td}_{y}^{\mathbb{C}^{*}}(X \hookrightarrow M) \in \widehat{H}_{*}^{\mathbb{C}^{*}}(M) \otimes \mathbb{Q}[y] \simeq \mathbb{Q}[[t]][y]
$$

is of the form

$$
\mathrm{eu}(M, 0) \cdot H\left(y, e^{-t}\right)
$$

where

- $\mathrm{eu}(M, 0)$ is the Euler class at the fixed point $p$, that is

$$
\mathrm{eu}(M, 0)=\left(\prod_{k=1}^{\operatorname{dim}(M)} w_{k}\right) t^{\operatorname{dim}(M)}
$$

is the product of the weights $w_{k} \in \mathbb{Z}$ of the action of $\mathbb{C}^{*}$ on $T_{0} M \simeq M$.

- $H(y, T)$ is a rational function in $T=e^{-t}$. When multiplied by

$$
\prod_{k=1}^{\operatorname{dim}(M)}\left(1-T^{w_{k}}\right)
$$

it is a polynomial in $T$ and $y$. The function $H(y, T)$ does not depend on the embedding.

We stress that the equivariant Hirzebruch class is an invariant of a singularity computed via resolution. That is so in general, but of course in some particular cases one can avoid resolutions - see our computation of the Hirzebruch class for Du Val singularities given in $§ 5.1$. 
On the other hand, we have a purely algebraic invariant of the representation of $G$. The (extended) Molien series is defined by the formula:

$$
\operatorname{Mol}(v, T)=\frac{1}{|G|} \sum_{k=0}^{\infty} \sum_{\ell=0}^{n} \operatorname{dim}\left(\left(\Lambda^{\ell}\left(\mathbb{C}^{n}\right)^{*} \otimes \operatorname{Sym}^{k}\left(\mathbb{C}^{n}\right)^{*}\right)^{G}\right) v^{\ell} T^{k}
$$

By Molien's theorem (1897)

$$
\operatorname{Mol}(0, T)=\frac{1}{|G|} \sum_{g \in G} \frac{1}{\operatorname{det}(1-T g)}
$$

An easy generalization (see Appendix 9) of Molien's theorem provides the formula

$$
\operatorname{Mol}(v, T)=\frac{1}{|G|} \sum_{g \in G} \frac{\operatorname{det}(1+v g)}{\operatorname{det}(1-T g)}
$$

The goal of this paper is to show a relation between the local equivariant Hirzebruch class of the quotient singularity $\mathbb{C}^{n} / G$ and the Molien series of (the chosen representation of) $G$. We prove

Theorem 1. For any quotient singularity we have

$$
\operatorname{td}_{y}^{\mathbb{C}^{*}}(X \hookrightarrow M)=\mathrm{eu}(M, 0) \operatorname{Mol}(y T, T),
$$

i.e.,

$$
H(y, T)=\operatorname{Mol}(y T, T) .
$$

This kind of interpretation of the local Hirzebruch class (or rather the Todd class for $y=0$ ) has appeared already in [Bau82, (3.8)] in a slightly different context.

The equality can be understood as a form of the McKay correspondence: a relation between geometry of the resolution of the quotient singularity and algebraic properties of the action. We prove Theorem 1 by applying the LefschetzRiemann-Roch theorem. A similar LRR-type formula for the elliptic genus was proved by Borisov and Libgober [BL05]. Their formula applies to crepant resolutions of global quotients of projective manifolds. Our approach is local. By the result of Borisov and Libgober specialized to the Hirzebruch class of a crepant resolution of $f: \widetilde{X} \rightarrow \mathbb{C}^{n} / G$ we obtain that

$$
\frac{f_{*} \operatorname{td}_{y}^{\mathbb{C}^{*}}(\tilde{X})}{\operatorname{eu}(M, 0)}=\sum_{h \in \operatorname{Conj}(G)}(-y)^{\operatorname{age}(h)} \operatorname{Mol}\left(C(h),\left(\mathbb{C}^{n}\right)^{h} ; y T, T\right),
$$

where $\operatorname{Mol}\left(C(h),\left(\mathbb{C}^{n}\right)^{h} ; y T, T\right)$ is the Molien series of the representation of the centralizer $C(h)$ on the space of fixed points $\left(\mathbb{C}^{n}\right)^{h}$. By age $(h)$ we understand $\sum_{k=1}^{n} \lambda_{k}$, where $e^{2 \pi i \lambda_{k}}, k=1, \ldots, n$ are the eigenvalues of $h$ and $\lambda_{k} \in[0,1) \cap \mathbb{Q}$.

Remark 2. The elliptic genus is more general than the Hirzebruch $\chi_{y}$-genus. The elliptic characteristic class specializes to $\operatorname{td}_{y}$ by a limit process. It might be interesting to see what is an interpretation of the local elliptic class from the representation theory point of view. 


\section{Lefschetz-Riemann-Roch}

First let us recall results of [BFQ79] which lead to a formula for the Todd class of the quotient variety $\operatorname{td}_{y=0}(Y / G)$. After that we review [CMSS12], adapting the notation to our purposes. These strengthened versions of Lefschetz-RiemannRoch allows us to compute the full Hirzebruch class. The proof of Theorem 1 is just checking that the methods of [BFQ79] and [CMSS12] apply in the equivariant case and interpreting the result for $Y=\mathbb{C}^{n}$.

\subsection{LRR for the Todd class}

Suppose $Y$ is a smooth quasiprojective variety on which a finite group $G$ acts. Set

$$
Y^{g}=\{x \in Y \mid g x=x\}
$$

Let $V \subset Y^{g}$ be a connected component. We will define a certain element $\lambda_{V}^{g} \in$ $K(V) \otimes \mathbb{C}$ in the $K$-theory of coherent sheaves. Let $N_{Y / V}^{*}$ be the conormal bundle

$$
\lambda_{V}^{g}=\sum_{a \text { root of unity }} \sum_{k=0}^{\operatorname{codim}(V)}(-1)^{k} a \cdot\left(\Lambda^{k} N_{Y / V}^{*}\right)_{g, a} \in K(V) \otimes \mathbb{C}
$$

where $\left(\Lambda^{k} N_{Y / V}^{*}\right)_{g, a} \in K(V)$ is the subbundle of $\Lambda^{k} N_{Y / V}^{*}$ on which $g$ acts with eigenvalue $a$. If $N_{Y / V}=\bigoplus_{k=1}^{\operatorname{codim}(V)} L_{k}$ was a direct sum of line bundles with $g$ acting on $L_{k}$ via the multiplication by $a_{k}(g)$ then we would have

$$
\lambda_{V}^{g}=\prod_{k=1}^{\operatorname{codim}(V)}\left(1-a_{k}(g)^{-1}\left[L_{k}\right]\right)
$$

By [Don69, Lem. 4.3] this element is invertible. Let

$$
L^{(g)} Y=\sum_{V \text { component of } Y^{g}}\left(\lambda_{V}^{g}\right)^{-1} \in K\left(Y^{g}\right) \otimes \mathbb{C} .
$$

Denote by $X=Y / G$ the quotient variety. By $[\mathrm{BFQ79}, \S 4(2)]$ we have an equality

$$
\left[\mathcal{O}_{X}\right]=\frac{1}{|G|} \sum_{g \in G} \pi_{*}^{g}\left(L^{(g)} Y\right) \in K(X) \otimes \mathbb{C}
$$

where $\pi^{g}: Y^{g} \rightarrow Y / G=X$ is the projection of the fixed point set to the quotient. The Todd class is obtained by applying the Grothendieck-RiemannRoch transformation

$$
\begin{aligned}
K(M) & \rightarrow H^{*}(M), \\
\mathcal{F} & \mapsto \operatorname{td}(M) \operatorname{ch}(\mathcal{F}),
\end{aligned}
$$


to formula (4). Here $\operatorname{td}(Y)$ is the classical Todd class and $\operatorname{ch}(-)$ is the Chern character. We obtain

$$
\begin{aligned}
\operatorname{td}(M) \operatorname{ch}\left(\mathcal{O}_{X}\right) & =\operatorname{td}(M) \operatorname{ch}\left(\frac{1}{|G|} \sum_{g \in G} \pi_{*}^{g}\left(L^{(g)} Y\right)\right) \\
& \stackrel{G R R}{=} \frac{1}{|G|} \sum_{g \in G} \pi_{*}^{g}\left(\operatorname{td}\left(Y^{g}\right) \operatorname{ch}\left(L^{(g)} Y\right)\right) .
\end{aligned}
$$

This is an expression for the image of Baum-Fulton-MacPherson class in $H^{*}(M)$, which coincides for rational singularities with $\operatorname{td}_{y=0}(X \hookrightarrow M)$ (see [BSY10, Example 3.2] or [Web16a, §14]). Each morphism $\pi^{g}$ can be factorized as $\pi \circ \iota^{g}$, where $\iota^{g}: Y^{g} \rightarrow Y$ is the inclusion of the fixed point set. We write

$$
\operatorname{td}_{y=0}(X \hookrightarrow M)=\frac{1}{|G|} \pi_{*} \sum_{g \in G} \iota_{*}^{g}\left(\operatorname{td}\left(Y^{g}\right) \operatorname{ch}\left(L^{(g)} Y\right)\right) .
$$

The class $\operatorname{ch}\left(L^{(g)} Y\right)$ is the sum of contributions $\left.\operatorname{ch}\left(\left(\lambda_{V}^{g}\right)^{-1}\right)\right)$ coming from various components of $Y^{g}$ and the expression for $\iota_{*}^{g}\left(\operatorname{td}\left(Y^{g}\right) \operatorname{ch}\left(\left(\lambda_{V}^{g}\right)^{-1}\right)\right)$ in terms of the Chern roots is the following:

$$
\begin{aligned}
\iota_{*}^{g}\left(\operatorname{td}\left(Y^{g}\right)\right. & \left.\cdot \prod_{k=1}^{\operatorname{codim}(V)} \frac{1}{1-a_{k}(g)^{-1} e^{-x_{k}}}\right) \\
& =\iota_{*}^{g}\left(\prod_{\ell=\operatorname{codim}(V)+1}^{\operatorname{dim}(Y)} \frac{1}{1-e^{-x_{\ell}}} \cdot \prod_{k=1}^{\operatorname{codim}(V)} \frac{1}{1-a_{k}(g)^{-1} e^{-x_{k}}}\right) \\
& =\prod_{k=1}^{\operatorname{codim}(V)} x_{k} \cdot \prod_{\ell=1+\operatorname{codim}(V)}^{\operatorname{dim}(Y)} \frac{x_{\ell}}{1-e^{-x_{\ell}}} \cdot \prod_{k=1}^{\operatorname{codim}(V)} \frac{1}{1-a_{k}(g)^{-1} e^{-x_{k}}},
\end{aligned}
$$

where

- $x_{k}$ for $k=1, \ldots, \operatorname{codim}(V)$ are the roots of $\left(N_{Y / V}\right)$,

- the eigenvalue corresponding to $x_{k}$ is $a_{k}(g)$,

- $x_{\ell}$ for $\ell=\operatorname{codim}(V), \ldots, \operatorname{dim}(Y)$ are the roots of $T_{V}$.

Finally we can write

$$
\iota_{*}^{g}\left(\operatorname{td}\left(Y^{g}\right) \operatorname{ch}\left(\left(\lambda_{V}^{g}\right)^{-1}\right)\right)=\prod_{k=1}^{\operatorname{dim}(Y)} \frac{x_{k}}{1-a_{k}(g)^{-1} e^{-x_{k}}}
$$

setting $a_{k}(g)=1$ for $k>\operatorname{codim}(V)$.

\subsection{LRR for the Hirzebruch class}

The same argument can be carried on for the full Hirzebruch class, as it is done in [CMSS12, Thm. 5.1] $]^{1}$. For simplicity let us assume that $Y^{g}$ has one component. We obtain

$$
\operatorname{td}_{y}(X \hookrightarrow M)=\frac{1}{|G|} \sum_{g \in G} \pi_{*}^{g}\left(\operatorname{td}_{y}\left(Y^{g}\right) \prod_{\theta \in(0,2 \pi)} \widetilde{T}_{y}^{\theta}\left(\left(N_{Y / Y^{g}}\right)_{g, e^{i \theta}}\right)\right),
$$

\footnotetext{
${ }^{1}$ The authors of $[\mathrm{CMSS12}]$ use the notation $\widetilde{T}_{y}$ for our $\operatorname{td}_{y}$.
} 
where for $\theta \in[0,1)$ the entry $\widetilde{T}_{y}^{\theta}\left(\left(N_{Y / Y^{g}}\right)_{g, e^{i \theta}}\right) \in H^{*}\left(Y^{g}\right) \otimes \mathbb{C}[y]$ is expressed in terms of $z_{s}$, the Chern roots of $\left(N_{Y / Y^{g}}\right)_{g, e^{i \theta}}$ as follows:

$$
\begin{aligned}
\widetilde{T}_{y}^{\theta}\left(\left(N_{Y / Y^{g}}\right)_{g, e^{i \theta}}\right) & =\prod_{s=1}^{\operatorname{dim}\left(\left(N_{\left.Y^{\prime} Y^{g}\right)_{g, e^{i \theta}}}\right.\right.} \frac{1+y e^{-z_{s}-i \theta}}{1-e^{-z_{s}-i \theta}} \\
& =\prod_{k: e^{i \theta}=a_{k}(g)} \frac{1+y a_{k}(g)^{-1} e^{-x_{k}}}{1-a_{k}(g)^{-1} e^{-x_{k}}}
\end{aligned}
$$

see [CMSS12, $\S 2.1$ (vi) and Def. 2.2]. The formula for $\operatorname{td}_{y}$ is almost the same as for $\widetilde{T}_{y}^{\theta}$ with $\theta=0$ :

$$
\operatorname{td}_{y}\left(Y^{g}\right)=\prod_{\ell=\operatorname{codim}(V)+1}^{\operatorname{dim}(Y)} \frac{x_{\ell}\left(1+y e^{-x_{\ell}}\right)}{1-e^{-x_{\ell}}}
$$

Therefore

$$
\begin{aligned}
& \iota_{*}^{g}\left(\operatorname{td}_{y}\left(Y^{g}\right) \prod_{\theta \in(0,2 \pi)} \widetilde{T}_{y}^{\theta}\left(\left(N_{Y / Y^{g}}\right)_{g, e^{i \theta}}\right)\right) \\
& \quad=\iota_{*}^{g}\left(\prod_{\ell=\operatorname{codim}(V)+1}^{\operatorname{dim}(Y)} \frac{x_{\ell}\left(1+y e^{-x_{\ell}}\right)}{1-e^{-x_{\ell}}} \cdot \prod_{k=1}^{\operatorname{codim}(V)} \frac{1+y a_{k}(g)^{-1} e^{-x_{k}}}{1-a_{k}(g)^{-1} e^{-x_{k}}}\right) \\
& =\prod_{k=1}^{\operatorname{codim}(V)} x_{k} \cdot \prod_{\ell=1+\operatorname{codim}(V)}^{\operatorname{dim}(Y)} \frac{x_{\ell}\left(1+y e^{-x_{\ell}}\right)}{1-e^{-x_{\ell}}} \cdot \prod_{k=1}^{\operatorname{codim}(V)} \frac{1+y a_{k}(g)^{-1} e^{-x_{k}}}{1-a_{k}(g)^{-1} e^{-x_{k}}} .
\end{aligned}
$$

As before, we write that class in a closed formula

$$
\iota_{*}^{g}\left(\operatorname{td}_{y}\left(Y^{g}\right) \prod_{\theta \in(0,2 \pi)} \widetilde{T}_{y}^{\theta}\left(\left(N_{Y / Y^{g}}\right)_{g, e^{i \theta}}\right)\right)=\prod_{k=1}^{\operatorname{dim}(Y)} \frac{x_{k}\left(1+y a_{k}(g)^{-1} e^{-x_{k}}\right)}{1-a_{k}(g)^{-1} e^{-x_{k}}}
$$

setting $a_{k}(g)=1$ for $k>\operatorname{codim}(V)$. We remark that the proof given in [CMSS12] uses $K$-theory of mixed Hodge modules of M. Saito [Sai00].

\subsection{Proof of Theorem 1}

Suppose that the group $\mathbb{C}^{*}$ acts on $Y$ and the action of $\mathbb{C}^{*}$ commutes with the action of $G$. Then $X=Y / G$ admits an action of $\mathbb{C}^{*}$ such that the projection

$$
\pi: Y \rightarrow X
$$

is $\mathbb{C}^{*}$-equivariant. We claim that formula (7) holds in equivariant cohomology. To justify that let us recall the definition of the equivariant Hirzebruch class via approximation [Web16a, Def. 7.1]:

$$
\operatorname{td}_{y}^{\mathbb{C}^{*}}(X)=\lim _{m \rightarrow \infty} p_{m}^{*}\left(\operatorname{td}_{y}\left(B_{m}\right)^{-1}\right) \cap \operatorname{td}_{y}\left(E_{m} \times \mathbb{C}^{*} X\right),
$$


where $E_{m} \rightarrow B_{m}$ is an approximation of the universal $\mathbb{C}^{*}$-bundle with $B_{m}$ being a smooth algebraic variety ${ }^{2}$ and $p_{m}: E_{m} \times \mathbb{C}^{*} Y \rightarrow B_{n}$ the associated approximation of the Borel construction. The group $G$ acts on $E_{m} \times \mathbb{C}^{*} Y$ and by the functoriality of the Riemann-Roch transformation we obtain the generalization of formula (7) for equivariant homology

$$
\operatorname{td}_{y}^{\mathbb{C}^{*}}(X)=\frac{1}{|G|} \sum_{g \in G} \pi_{*} \iota_{*}^{g}\left(\operatorname{td}_{y}^{\mathbb{C}^{*}}\left(Y^{g}\right) \prod_{\theta \in(0,2 \pi)} \widetilde{T}_{y}^{\theta}\left(\left(N_{Y / Y^{g}}\right)_{g, e^{i \theta}}\right)\right) \in \widehat{H}_{\mathbb{C}^{*}, *}(X) \otimes \mathbb{C}[y]
$$

Here the equivariant class $\iota_{*}^{g}\left(\operatorname{td}_{y}^{\mathbb{C}^{*}}\left(Y^{g}\right) \prod_{\theta \in(0,2 \pi)} \widetilde{T}_{y}^{\theta}\left(\left(N_{Y / Y^{g}}\right)_{g, e^{i \theta}}\right)\right)$ is given by the same formula (6), which is valid in the equivariant cohomology of $Y^{g}$. Since $X$ has only quotient singularities, we have Poincaré duality

$$
\widehat{H}_{T, *}(X) \simeq \widehat{H}_{\mathbb{C}^{*}}^{2 \operatorname{dim}(X)-*}(X)
$$

Let $G \subset \mathrm{GL}_{n}(\mathbb{C})$ be a finite group and let $Y=\mathbb{C}^{n}$ be the natural representation of $G$. Assume $Y^{G}=\{0\}$. The scalar action of $\mathbb{C}^{*}$ commutes with the action of $G$. We apply formula (10) to computing the equivariant Hirzebruch class. As before, each morphism $\pi^{g}$ is factorized as $\pi \circ \iota^{g}$, where $\iota^{g}:\left(\mathbb{C}^{n}\right)^{g} \rightarrow \mathbb{C}^{n}$ is the inclusion. Let us compute the equivariant version of the class computed in (6):

$$
\begin{aligned}
& \iota_{*}^{g}\left(\operatorname{td}_{y}^{\mathbb{C}^{*}}\left(Y^{g}\right) \prod_{\theta \in(0,2 \pi)} \widetilde{T}_{y}^{\theta}\left(\left(N_{Y / Y^{g}}\right)_{g, e^{i \theta}}\right)\right) \\
& \quad=t^{\operatorname{codim}\left(\left(\mathbb{C}^{n}\right)^{g}\right)} \prod_{\ell=1}^{\operatorname{dim}\left(\left(\mathbb{C}^{n}\right)^{g}\right)} \frac{t\left(1+y e^{-t}\right)}{1-e^{-t}} \cdot \prod_{k=1}^{\operatorname{codim}\left(\left(\mathbb{C}^{n}\right)^{g}\right)} \frac{\left(1+y a_{k}(g)^{-1} e^{-t}\right)}{1-a_{k}(g)^{-1} e^{-t}} .
\end{aligned}
$$

The numbers $a_{k}(g) \in \mathbb{C}$ for $k=1, \ldots, \operatorname{codim}\left(\left(\mathbb{C}^{n}\right)^{g}\right)$ are the eigenvalues of $g$ which are different from 1 . Finally, we can write

$$
\iota_{*}^{g}\left(\operatorname{td}_{y}^{\mathbb{C}^{*}}\left(Y^{g}\right) \prod_{\theta \in(0,2 \pi)} \widetilde{T}_{y}^{\theta}\left(\left(N_{Y / Y^{g}}\right)_{g, e^{i \theta}}\right)\right)=t^{n} \prod_{k=1}^{n} \frac{1+y a_{k}(g)^{-1} T}{1-a_{k}(g)^{-1} T}
$$

where $a_{k}(g)$ for $k=1, \ldots, n$ are all the eigenvalues of $g$ acting on $\mathbb{C}^{n}$ and $T=e^{-t}$. We assume that the ambient space $M$ containing $X$ is another vector space. Let $j: X \rightarrow M$ be the inclusion. For any element $\alpha \in H_{\mathbb{C}^{*}}^{*}(Y)$ we have

$$
\frac{\left.\left(j_{*} \pi_{*} \alpha\right)\right|_{0}}{\mathrm{eu}(M, 0)}=\frac{\left.\alpha\right|_{0_{Y}}}{\mathrm{eu}(Y, 0)} \in \widehat{H}_{\mathbb{C}^{*}}^{*}(p t) \otimes \mathbb{C}\left[y, t^{-1}\right] \simeq \mathbb{C}[[t]] \otimes \mathbb{C}\left[y, t^{-1}\right]
$$

Setting $\alpha=\iota_{*}^{g}\left(\operatorname{td}_{y}^{\mathbb{C}^{*}}\left(Y^{g}\right) \prod_{\theta \in(0,2 \pi)} \widetilde{T}_{y}^{\theta}\left(\left(N_{Y / Y^{g}}\right)_{g, e^{i \theta}}\right)\right)$ and summing over the elements of $g$ we obtain the expression for $\left.\operatorname{td}_{y}^{\mathbb{C}^{*}}(X \hookrightarrow M)\right|_{0} / \mathrm{eu}(M, 0)$. We change the

\footnotetext{
${ }^{2}$ The standard model for $B_{m}$ is $\mathbb{P}^{m}$ and $E_{m}=\mathbb{C}^{m+1} \backslash\{0\}$.
} 
order of the summation replacing $g$ by $g^{-1}$ and we conclude that

$$
\begin{aligned}
\frac{\left.\operatorname{td}_{y}^{\mathbb{C}^{*}}(X \hookrightarrow M)\right|_{0}}{\operatorname{eu}(M, 0)} & =\frac{\left.j_{*} \operatorname{td}_{y}^{\mathbb{C}^{*}}(X)\right|_{0}}{\operatorname{eu}(M, 0)} \\
& =\sum_{g \in G} \prod_{k=1}^{n} \frac{1+a_{k}(g) y T}{1-a_{k}(g) T}=\sum_{g \in G} \frac{\operatorname{det}(1+y T g)}{\operatorname{det}(1-T g)}
\end{aligned}
$$

By formula (2) this is exactly the expression for the extended Molien series with $v=y T$. Hence

$$
\left.\operatorname{td}_{y}^{\mathbb{C}^{*}}(X \hookrightarrow M)\right|_{0}=\mathrm{eu}(M, 0) \operatorname{Mol}(v T, T) .
$$

The restriction to 0 is an isomorphism on equivariant cohomology since $M$ is contractible. Thus we obtain the claim.

\section{The functional equation}

The function

$$
H(y, T)=\frac{1}{\operatorname{eu}(M, 0)} \operatorname{td}_{y}^{\mathbb{C}^{*}}\left(\mathbb{C}^{n} / G \hookrightarrow M\right)
$$

has some symmetries.

\subsection{General linear group}

The basic symmetry holds for arbitrary $G \subset \mathrm{GL}_{n}(\mathbb{C})$; further symmetries appear for subgroups of $\mathrm{SL}_{n}(\mathbb{C})$ or $\mathrm{Sp}_{n}(\mathbb{C})$.

Proposition 3 (Duality). For any $n$-dimensional quotient singularity we have

$$
H(1 / y, 1 / T)(-y)^{n}=H(y, T)
$$

Proof.

$$
\begin{aligned}
H(1 / y, 1 / T) & =\operatorname{Mol}(1 /(y T), 1 / T)=\frac{1}{|G|} \sum_{g \in G} \prod_{k=1}^{n} \frac{1+a_{k}(g)(y T)^{-1}}{1-a_{k}(g) T^{-1}} \\
& =\frac{1}{|G|} \sum_{g \in G} \prod_{k=1}^{n} \frac{a_{k}(g)(y T)^{-1}\left(y T a_{k}(g)^{-1}+1\right)}{a_{k}(g) T^{-1}\left(T a_{k}(g)^{-1}-1\right)} \\
& =\frac{1}{|G|(-y)^{n}} \sum_{g \in G} \prod_{k=1}^{n} \frac{1+y T a_{k}(g)^{-1}}{1-T a_{k}(g)^{-1}}
\end{aligned}
$$

We replace $g$ by $g^{-1}$ in the summation and we note that the eigenvalues of $g^{-1}$ are inverses of the eigenvalues of $g$ :

$$
\begin{aligned}
H(1 / y, 1 / T) & =\frac{1}{|G|(-y)^{n}} \sum_{g \in G} \prod_{k=1}^{n} \frac{1+y T a_{k}\left(g^{-1}\right)}{1-T a_{k}\left(g^{-1}\right)} \\
& =(-y)^{-n} \operatorname{Mol}(y T, T)=(-y)^{-n} H(y, T) .
\end{aligned}
$$


This duality does not hold for arbitrary singularities. For example, for the affine cone over a curve of degree 4 in $\mathbb{P}^{2}$ we have

$$
H(y, T)=2(1+y) \frac{T+T^{2}+\left(3 T-T^{2}\right) y}{(1-T)^{2}}+1
$$

and the duality does not hold. (The formula for the Hirzebruch classes of affine cones is given in [Web16a, Prop. 10.3].)

Proposition 4 (Divisibility). For any finite subgroup $G \subset \mathrm{GL}_{n}(\mathbb{C})$ the polynomial $H(y, T)-1 \in \mathbb{Q}(T)[y]$ is divisible by $y+1$.

Proof. We have

$$
H(y, T)-1=\operatorname{Mol}(y T, T)-1=\frac{1}{|G|} \sum_{g \in G} \frac{\operatorname{det}(1+y T g)-\operatorname{det}(1-T g)}{\operatorname{det}(1-T g)} .
$$

The expression vanishes for $y=-1$, so divisibility in $\mathbb{Q}(T)[y]$ follows.

Divisibility can be explained geometrically by the fact that

$$
\mathrm{eu}(M, 0)(H(y, T)-1)=\operatorname{td}_{y}^{\mathbb{C}^{*}}\left(\left(\mathbb{C}^{n} \backslash\{0\}\right) / G \hookrightarrow M\right)
$$

and $\left(\mathbb{C}^{n} \backslash\{0\}\right) / G$ is a sum of nonconstant orbits of $\mathbb{C}^{*}$. Each orbit is isomorphic to $\mathbb{C}^{*}$ and $\chi_{y}\left(\mathbb{C}^{*}\right)=-(y+1)$. The divisibility follows from the multiplicative properties of $\chi_{y}$-genus and the Hirzebruch class.

\subsection{Special linear group}

Proposition 5 (SL-duality). For any finite subgroup $G \subset \mathrm{SL}_{n}(\mathbb{C})$

$$
H(y, T)=\frac{H\left(y T^{2}, 1 / T\right)}{(-T)^{n}} .
$$

Proof. First note that for $G \subset \mathrm{SL}_{n}(\mathbb{C})$ the sequence of exterior powers is symmetric, i.e., $\Lambda^{l}\left(\mathbb{C}^{n}\right) \simeq \Lambda^{n-l}\left(\mathbb{C}^{n}\right)$. Hence we have

$$
\operatorname{Mol}(v, T)=\operatorname{Mol}(1 / v, T) v^{n} .
$$

Then, applying Prop. 3, we obtain

$$
\begin{aligned}
\frac{H\left(y T^{2}, 1 / T\right)}{(-T)^{n}} & =\frac{\operatorname{Mol}(y T, 1 / T)}{(-T)^{n}}=\frac{\operatorname{Mol}(1 /(y T), 1 / T)}{(-T)^{n}} \cdot y^{n} T^{n} \\
& =H(1 / y, 1 / T)(-y)^{n}=\frac{H(y, T)}{(-y)^{n}} \cdot(-y)^{n}=H(y, T) .
\end{aligned}
$$

The SL-duality means that the coefficients of $H(y, T)$ as a polynomial in $y$ are palindromic with respect to $T$. This kind of duality does not hold in general. For example, for the quotient of $\mathbb{C}^{2}$ by $\mathbb{Z}_{n}$ acting diagonally we have

$$
H(y, T)=\frac{1+(n-1) T^{n}+2 n T^{n} y+\left((n-1) T^{n}+T^{2 n}\right) y^{2}}{\left(1-T^{n}\right)^{2}} .
$$




\subsection{Symplectic group}

Proposition 6 (Symplectic divisibility). If $G \subset \mathrm{Sp}_{n}(\mathbb{C}) \subset \mathrm{SL}_{2 n}(\mathbb{C})$ is a finite symplectic group then $H(y, T)-(-y)^{n}$ is divisible by $y T^{2}+1$.

Proof. We have

$$
\begin{aligned}
H(y, T)-(-y)^{n} & =\operatorname{Mol}(y T, T)-(-y)^{n} \\
& =\frac{1}{|G|} \sum_{g \in G} \frac{\operatorname{det}(1+y T g)-(-y)^{n} \operatorname{det}(1-T g)}{\operatorname{det}(1-T g)},
\end{aligned}
$$

so it suffices to prove that for any $g \in G$ the polynomial

$$
P(y, T)=\operatorname{det}(1+y T g)-(-y)^{n} \operatorname{det}(1-T g)
$$

is divisible by $\left(y T^{2}+1\right)$. If we view it as a polynomial in $y$ over the field of rational functions $\mathbb{Q}(T)$, we need only to show that $P\left(-\frac{1}{T^{2}}, T\right)=0$. One looks at

$$
P\left(-\frac{1}{T^{2}}, T\right)=\operatorname{det}\left(1-g \frac{1}{T}\right)-\frac{1}{T^{2 n}} \operatorname{det}(1-T g)
$$

which is 0 if and only if the coefficients $d_{0}, \ldots, d_{2 n}$ of the polynomial $\operatorname{det}(1-T g)$ form a symmetric sequence: $d_{k}=d_{2 n-k}$ for any $k \in\{0, \ldots, n\}$. Equivalently, the sequence of eigenvalues $\left(a_{1}(g), \ldots, a_{2 n}(g)\right)$ of $g$ is a permutation of the sequence $\left(a_{1}(g)^{-1}, \ldots, a_{2 n}(g)^{-1}\right)$. And symplectic matrix groups have this property.

Corollary 7. The polynomial $H(y, T)-(-y)^{n}$ for a symplectic quotient $\mathbb{C}^{2 n} / G$ is divisible by $(y+1)\left(y T^{2}+1\right)$.

Proof. Observe that $(-y)^{n}-1$ is divisible by $y+1$ and use Propositions 4 and 6 .

Corollary 8. For a surface quotient singularity the polynomial $H(y, T)$ is determined by $H(0, T)$. It is of the form

$$
(y+1)(f(T)+y f(1 / T))+1
$$

where $f(T)=H(0, T)-1$. For symplectic quotients the Hirzebruch class is equal to

$$
H(y, T)=(y+1)\left(y T^{2}+1\right) H(0, T)-y .
$$

Proof. The first statement follows from Propositions 3 and 4 since for a surface singularity $H(y, T)$ is of degree two as a polynomial in $y$. The second statement follows from Corollary 7. 


\section{Hirzebruch class of quotient surfaces}

\subsection{Du Val singularities as hypersurfaces}

The Hirzebruch classes of Du Val singularities, i.e., the symplectic quotients of $\mathbb{C}^{2}$, are surprisingly simple. All of these quotients can be realized as hypersurfaces $X \subset \mathbb{C}^{3}$ given by quasihomogeneous polynomials. In general, for a hypersurface in a smooth variety $X \subset M$ the image of the Baum-Fulton-MacPherson class in $H^{*}(M)$ is given by the formula

$$
\operatorname{td}(M) \operatorname{ch}\left(\mathcal{O}_{X}\right)=\operatorname{td}(M)\left(1-e^{-[X]}\right),
$$

see $\left[\right.$ Ful98, 18.3.5]. The embedding into $\mathbb{C}^{3}$ can be made equivariant and the formula (13) holds for equivariant classes. Since the Baum-Fulton-MacPherson class coincides with the class $\operatorname{td}_{y=0}^{\mathbb{C}^{*}}$, we obtain

$$
H(0, T)=\frac{\operatorname{td}^{\mathbb{C}^{*}}\left(\mathbb{C}^{3}\right)}{\mathrm{eu}\left(\mathbb{C}^{3}, 0\right)} \operatorname{ch}^{\mathbb{C}^{*}}\left(\mathcal{O}_{X}\right)=\frac{1-T^{d}}{\left(1-T^{w_{1}}\right)\left(1-T^{w_{2}}\right)\left(1-T^{w_{3}}\right)},
$$

where $w_{i}$ are the weights of the action of $\mathbb{C}^{*}$ on $\mathbb{C}^{3}$ and $d$ is the weighted degree of the polynomial defining the hypersurface. By Corollary 8 we have

$$
H(y, T)=(y+1)\left(y T^{2}+1\right) \frac{1-T^{d}}{\left(1-T^{w_{1}}\right)\left(1-T^{w_{2}}\right)\left(1-T^{w_{3}}\right)}-y .
$$

\subsection{Hirzebruch classes of Du Val singularities}

We list below the Hirzebruch classes for the series A, D and E:

- Group $\mathbb{Z}_{n}$, singularity $\mathrm{A}_{n-1}, x^{n}+y^{2}+z^{2}$

$$
(y+1)\left(y T^{2}+1\right) \frac{1-T^{2 n}}{\left(1-T^{2}\right)\left(1-T^{n}\right)^{2}}-y .
$$

By Theorem 1 this expression is equal to

$$
\frac{1}{n} \sum_{k=0}^{n-1} \frac{1+2 \cos (2 k \pi / n) y T+y^{2} T^{2}}{1-2 \cos (2 k \pi / n) T+T^{2}} .
$$

- Binary dihedral group $B D_{4(n-2)}$, singularity $\mathrm{D}_{n}, x^{n-1}+y^{2} x+z^{2}$

$$
(y+1)\left(y T^{2}+1\right) \frac{1-T^{4 n-4}}{\left(1-T^{4}\right)\left(1-T^{2(n-2)}\right)\left(1-T^{2(n-1)}\right)}-y .
$$

- Binary tetrahedral group $B T_{24}$, singularity $\mathrm{E}_{6}, x^{4}+y^{3}+z^{2}$

$$
(y+1)\left(y T^{2}+1\right) \frac{1-T^{24}}{\left(1-T^{6}\right)\left(1-T^{8}\right)\left(1-T^{12}\right)}-y .
$$


- Binary octahedral group $B O_{48}$, singularity $\mathrm{E}_{7}, x^{3}+x y^{3}+z^{2}$

$$
(y+1)\left(y T^{2}+1\right) \frac{1-T^{36}}{\left(1-T^{12}\right)\left(1-T^{8}\right)\left(1-T^{18}\right)}-y .
$$

- Binary icosahedral group $B I_{60}$, singularity $\mathrm{E}_{8}, x^{5}+y^{3}+z^{2}$

$$
(y+1)\left(y T^{2}+1\right) \frac{1-T^{60}}{\left(1-T^{12}\right)\left(1-T^{20}\right)\left(1-T^{30}\right)}-y .
$$

The Chern-Schwartz-MacPherson classes are specializations of Hirzebruch classes: $c^{S M} / \mathrm{eu}(M, 0)=\lim _{y \rightarrow-1} H\left(y, e^{-(1+y) t}\right)$. The local equivariant version of Chern-Schwartz-MacPherson classes is studied in [Web12]. Here are the formulas for Du Val singularities.

$$
\begin{array}{ccccc}
\mathrm{A}_{n-1} & \mathrm{D}_{n} & \mathrm{E}_{6} & \mathrm{E}_{7} & \mathrm{E}_{8} \\
\frac{n t^{2}+2 t+1}{n t^{2}}, & \frac{4(n-2) t^{2}+2 t+1}{4(n-2) t^{2}}, & \frac{24 t^{2}+2 t+1}{24 t^{2}}, & \frac{48 t^{2}+2 t+1}{48 t^{2}}, & \frac{120 t^{2}+2 t+1}{120 t^{2}} .
\end{array}
$$

\subsection{Hirzebruch class of surface singularities via resolution}

Suppose that $(S, 0) \subset(M, 0)$ is a germ of isolated surface singularity embedded in a smooth variety. Suppose a torus $\mathbb{C}^{*}$ acts on $M$ preserving $S$ and 0 . As before, $M$ can be taken as a vector space with a linear action of $\mathbb{C}^{*}$. Let $\widetilde{S} \rightarrow S \subset M$ be an equivariant resolution of $S$ with the exceptional divisor having simple normal crossings. By functoriality and additivity of the Hirzebruch class we have

$$
\begin{aligned}
\operatorname{td}_{y}^{\mathbb{C}^{*}}(S \hookrightarrow M) & =p_{*} \operatorname{td}_{y}^{\mathbb{C}^{*}}(\widetilde{S})-p_{*} \operatorname{td}_{y}^{\mathbb{C}^{*}}(E \hookrightarrow \widetilde{S})+\operatorname{td}_{y}^{\mathbb{C}^{*}}(0 \hookrightarrow M) \\
& =p_{*} \operatorname{td}_{y}^{\mathbb{C}^{*}}(\widetilde{S})+\left(1-\chi_{y}(E)\right)[0]
\end{aligned}
$$

where $p: \widetilde{S} \rightarrow M$ is the resolution map composed with the embedding into $M, E=$ $\bigcup_{i=1}^{k} E_{i}$ is the exceptional divisor and $[0] \in H_{\mathbb{C}^{*}}^{4}(M)$ is the class of the point. The $\chi_{y}$-genus of $E$ can be computed by additivity:

$$
\chi_{y}(E)=\sum_{i=1}^{k} \chi_{y}\left(E_{i}\right)-\ell \chi_{y}(p t)=\sum_{i=1}^{k}\left(1-g_{i}\right)(1-y)-\ell
$$

where $g_{i}$ is the genus of $E_{i}$ and $\ell$ is the number of intersection points. If $E$ is a tree of rational curves then

$$
\chi_{y}(E)=-k(1-y)-(k-1)=-k y+1
$$

and

$$
\operatorname{td}_{y}^{\mathbb{C}^{*}}(S \hookrightarrow M)=p_{*} \operatorname{td}_{y}^{\mathbb{C}^{*}}(\widetilde{S})+k y[0]
$$


To compute the push forward $p_{*} \operatorname{td}_{y}^{\mathbb{C}^{*}}(\widetilde{S})$ one can apply Atiyah-Bott or BerlineVergne localization, [AB84], [BV82], which holds in the relative case by [PT07, Cor. 3.2]. If the action of $\mathbb{C}^{*}$ has only isolated fixed points, then

$$
\begin{aligned}
\left.p_{*} \operatorname{td}_{y}^{\mathbb{C}^{*}}(\widetilde{S})\right|_{0} & =\left.\operatorname{eu}(M, 0) \sum_{p \in \widetilde{S}^{\mathbb{C}^{*}}} \frac{1}{\operatorname{eu}(\widetilde{S}, p)} \operatorname{td}_{y}^{\mathbb{C}^{*}}(\widetilde{S})\right|_{p}= \\
& =\operatorname{eu}(M, 0) \sum_{p \in \widetilde{S}^{\mathbb{C}^{*}}} \frac{1+y T^{w_{1}(p)}}{1-T^{w_{1}(p)}} \frac{1+y T^{w_{2}(p)}}{1-T^{w_{2}(p)}},
\end{aligned}
$$

where $w_{i}(p)$ for $i=1,2$ are the weights of the $\mathbb{C}^{*}$ action on the tangent space $T_{p} \widetilde{S}$. If the fixed point set $\widetilde{S}^{\mathbb{C}^{*}}$ is not finite then the expression for the Hirzebruch class has an additional summand corresponding to each fixed component $E_{f i x}$

$$
\left.\int_{E_{f i x}} \frac{1}{c_{1}\left(N_{f i x}\right)} \operatorname{td}_{y}^{\mathbb{C}^{*}}(\widetilde{S})\right|_{E_{f i x}}=\int_{E_{f i x}} \operatorname{td}_{y}^{\mathbb{C}^{*}}\left(E_{f i x}\right) \frac{1+y e^{-c_{1}\left(N_{f i x}\right)}}{1-e^{-c_{1}\left(N_{f i x}\right)}},
$$

where $N_{f i x}$ is the normal bundle to the fixed component.

We will illustrate the computations by the example of $\mathrm{Du}$ Val singularities. Among Du Val singularities only $\mathrm{A}_{n}$ with $n$ even has isolated fixed points in the resolution. For the remaining $\mathrm{Du}$ Val singularities there always exists exactly one fixed component:

- the central component of $E$ for the series $\mathrm{A}_{n}$ with $n$ odd,

- the component which meets three other components for the series $D_{n}$ and $\mathrm{E}_{6}, \mathrm{E}_{7}, \mathrm{E}_{8}$.

To describe the situation we encode the weights in the Dynkin diagram: the edges, i.e., the intersections of divisors, are labelled by the weights of the action of the torus on the tangent space at the intersection point. The loose edges of the diagram correspond to the fixed points which are not the intersection points. Let us give a few examples:

- The singularity $A_{6}$

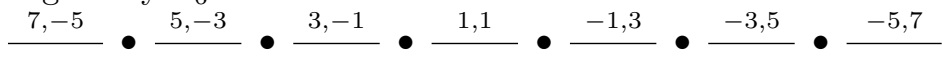

- The singularity $A_{5}$

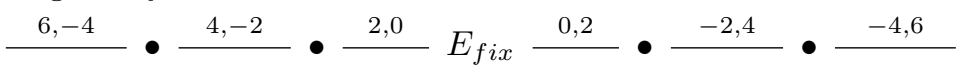

- The singularity $\mathrm{D}_{5}$

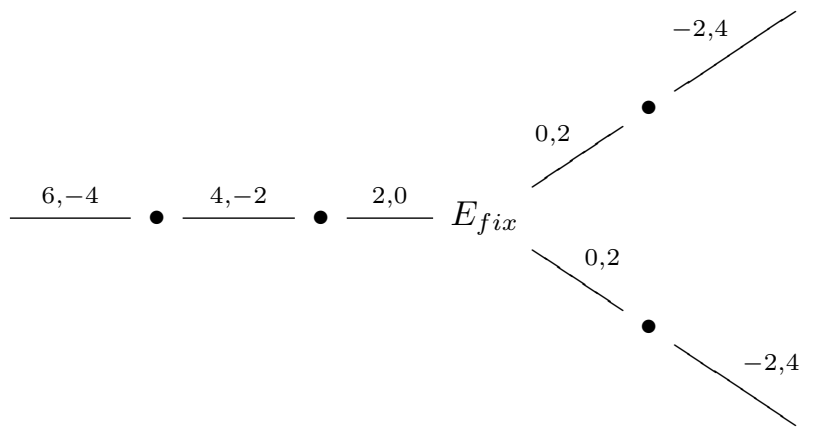


- The singularity $\mathrm{E}_{7}$

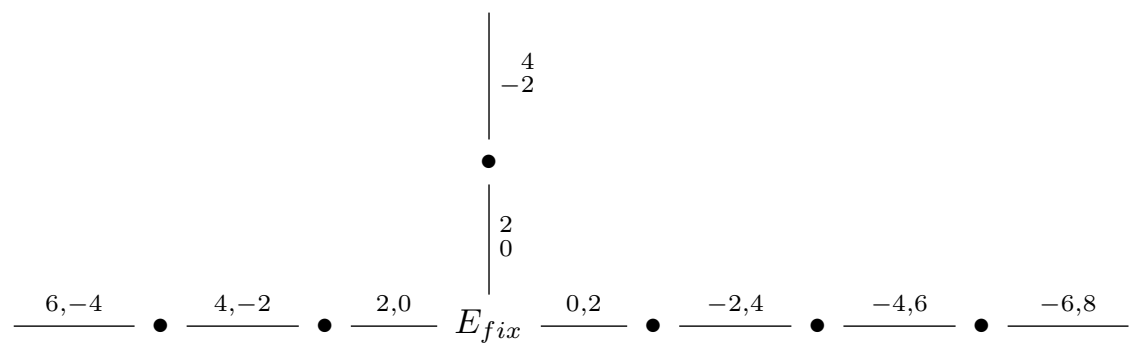

The weights are computed in the following way: $A_{n}$ admits an action of twodimensional torus, so it is an affine toric surface. The structure of the resolution can be read from the fan. The singularity $D_{n}$ is a quotient of $A_{2 n}$ by $\mathbb{Z}_{2}$ and the series $\mathrm{E}_{k}$ can be analyzed directly: the curve with three intersection points has to be fixed by $\mathbb{C}^{*}$ and the action on the remaining curves can be computed inductively: the action on the normal direction determines the self-intersection which is -2 .

The neighbourhood of the fixed component for Du Val singularities is equivariantly isomorphic to the resolution of $A_{1}$ singularity. The contribution of that component is equal to

$$
\frac{\operatorname{td}_{y}^{\mathbb{C}^{*}}\left(\mathbb{C}^{2} / \mathbb{Z}_{2} \hookrightarrow M\right)}{\mathrm{eu}(M, 0)}-y=\frac{(y+1)\left(y T^{2}+1\right)\left(T^{2}+1\right)}{\left(1-T^{2}\right)^{2}}-2 y
$$

by $\S 5.1$ or by a direct computation. For example, from the formula (14) and the diagram above we compute the Hirzebruch class for $E_{7}$ :

$$
\begin{aligned}
\frac{1}{\mathrm{eu}(M, 0)} p_{*} \operatorname{td}_{y}^{\mathbb{C}^{*}}(\widetilde{S})= & \frac{(y+1)\left(y T^{2}+1\right)\left(T^{2}+1\right)}{\left(1-T^{2}\right)^{2}}-2 y+3 \frac{\left(1+y T^{-2}\right)\left(1+T^{4} y\right)}{\left(1-T^{-2}\right)\left(1-T^{4}\right)} \\
& +2 \frac{\left(1+y T^{-4}\right)\left(1+T^{6} y\right)}{\left(1-T^{-4}\right)\left(1-T^{6}\right)}+\frac{\left(1+y T^{-6}\right)\left(1+T^{8} y\right)}{\left(1-T^{-6}\right)\left(1-T^{8}\right)} .
\end{aligned}
$$

After simplification we obtain

$$
\frac{(y+1)\left(y T^{2}+1\right)\left(1-T^{36}\right)}{\left(1-T^{12}\right)\left(1-T^{8}\right)\left(1-T^{18}\right)}-8 y .
$$

To get the formula for $\operatorname{td}_{y}^{\mathbb{C}^{*}}(S \hookrightarrow M) / \mathrm{eu}(M, 0)$ one has to add $7 y$.

\subsection{Relation with Poincaré series}

In [CDGZ04] there are constructed Poincaré series of surface singularities. They are generating series for multifiltrations in $\mathcal{O}_{X}$ defined by valuations in the components of the exceptional divisors of the minimal resolution. This filtration is related to the grading defined by the torus action. For the singularities $A_{2 m-1}, D_{n}, E_{6}, E_{7}$ and $E_{8}$ there is a component which is fixed by the torus. When we specialize the 
Poincaré series to that component we obtain the classical Molien series $(v=0)$ which coincides with $H(0, T)$.

- General form of Poincaré series for $\mathrm{A}_{n}$ singularity (notation from [CDGZ04, Ex. 1])

$$
\frac{1-\left(\prod_{k=1}^{n} t_{k}\right)^{n+1}}{\left(1-\prod_{k=1}^{n} t_{k}\right)\left(1-\prod_{k=1}^{n} t_{k}^{k}\right)\left(1-\prod_{k=1}^{n} t_{k}^{-k+n+1}\right)} .
$$

- For $n=2 m-1$ substituting $t_{m}=T^{2}$ and $t_{k}=1$ for $k \neq m$ we obtain the function $H(0, T)=\operatorname{Mol}(0, T)$.

- For the singularity $\mathrm{D}_{4}$ the Poincaré series ([CDGZ04, Ex. 2]) is

$$
\frac{\left(1-t_{1} t_{2} t_{3} t_{4}^{2}\right)\left(t_{1}^{2} t_{2}^{2} t_{3}^{2} t_{4}^{3}+1\right)}{\left(1-t_{1}^{2} t_{2} t_{3} t_{4}^{2}\right)\left(1-t_{1} t_{2}^{2} t_{3} t_{4}^{2}\right)\left(1-t_{1} t_{2} t_{3}^{2} t_{4}^{2}\right)} .
$$

When we substitute $t_{4}=T^{2}$ and $t_{i}=1$ for $i \neq 4$ we obtain the function $H(0, T)=\left(T^{6}+1\right) /\left(1-T^{4}\right)^{2}$.

- For $\mathrm{D}_{5}$ after the substitution $t_{3}=T^{2}$ and $t_{i}=1$ for $i \neq 3$ we obtain the function $H(0, T)=\left(T^{8}+1\right) /\left(1-T^{4}\right)\left(1-T^{6}\right)$.

- But there are more possible substitutions; for example, for $\mathrm{A}_{n}: t_{n}=t_{1}=T$ and $t_{k}=1$ for $k \neq 1, n$, which works also for any $n$.

- For $n=2 m: t_{m}=t_{m+1}=T$, and $t_{k}=1$ for $k \neq 1$.

The relation between the equivariant Hirzebruch class (or rather the equivariant Todd class) and the Poincaré series we will study elsewhere.

\subsection{Toric singularities}

If the group $G \subset \mathrm{GL}_{n}(\mathbb{C})$ is abelian, we may assume that the chosen representation is diagonal, so the action of $G$ commutes with the action of the torus $\mathbb{T}=\left(\mathbb{C}^{*}\right)^{n}$. The quotient singularity is an affine toric variety. Its local equivariant Hirzebruch class with respect to the action of the torus $\mathbb{T}$ can be computed via a toric resolution. By Brion-Vergne [BV97] the local Todd class (i.e., for $y=0$ ) can be computed by counting lattice points in the dual cone. In fact, the method of the proof in [BV97] is as in our case based on the localization to the fixed points, a version of Lefschetz-Riemann-Roch. The generalization of Brion-Vergne result for the Hirzebruch class is given in [Web16a].

Proposition 9. Let $X_{\sigma}$ be an affine toric variety given by the cone $\sigma$. Let $p$ be the fixed point of $X_{\sigma}$. Then

$$
\frac{\left.\operatorname{td}_{y}^{\mathbb{T}}\left(X_{\sigma}\right)\right|_{p}}{\operatorname{eu}(M, p)}=\sum_{\tau \subset \sigma^{\vee}}(1+y)^{\operatorname{dim}(\tau)} \sum_{m \in \operatorname{int}(\tau) \cap \Lambda} e^{-m} .
$$

Here we identify the lattice $\Lambda=\operatorname{Hom}\left(\mathbb{T}, \mathbb{C}^{*}\right)$ with $H_{\mathbb{T}}^{2}(p t ; \mathbb{Z})$ and the summation is taken with respect to faces (of any dimension) of the dual cone $\sigma^{\vee}$.

Remark 10. When we restrict the action to a one-dimensional diagonal torus then the formula above (at least when we set $y=0$ ) reduces to computation of the 
classical Molien series: counting lattice points corresponds to counting the dimensions of Sym* $\left(\mathbb{C}^{n}\right)^{G}$. This way we obtain another proof of Theorem 1 for diagonal representations.

Let us give an example of surface singularities with $G=\mathbb{Z}_{n} \subset \mathrm{SL}_{2}$ (C), i.e., $A_{n-1}$ with the action of $\left(\mathbb{C}^{*}\right)^{2}$. We set $T_{k}=e^{-t_{k}}$ for $k=1,2$. We have four ways of computing the Hirzebruch class and obtain four different expressions. We leave to the reader checking that these results are equal.

- The Hirzebruch class computed for $A_{n-1}$ as a hypersurface in $\mathbb{C}^{3}$ :

$$
(1+y)\left(1+T_{1} T_{2} y\right) \frac{1-\left(T_{1} T_{2}\right)^{n}}{\left(1-T_{1} T_{2}\right)\left(1-T_{1}^{n}\right)\left(1-T_{2}^{n}\right)}-y .
$$

- The Hirzebruch class via resolution:

$$
\sum_{i=0}^{n-1} \frac{1+y T_{1}^{i+1} T_{2}^{i+1-n}}{1-T_{1}^{i+1} T_{2}^{i+1-n}} \cdot \frac{1+y T_{1}^{-i} T_{2}^{n-i}}{1-T_{1}^{-i} T_{2}^{n-i}}+(n-1) y .
$$

- The Hirzebruch class via counting lattice points:

$$
1+(y+1)\left(\frac{T_{1}^{n}}{1-T_{1}^{n}}+\frac{T_{2}^{n}}{1-T_{2}^{n}}\right)+(y+1)^{2} \frac{1}{1-T_{1}^{n}} \frac{1}{1-T_{2}^{n}} \sum_{k=1}^{n}\left(T_{1} T_{2}\right)^{k} .
$$

- The Hirzebruch class via Lefschetz-Riemann-Roch:

$$
\frac{1}{n} \sum_{k=0}^{n-1} \frac{1+y e^{2 k \pi i / n} T_{1}}{1-e^{2 k \pi i / n} T_{1}} \cdot \frac{1+y e^{-2 k \pi i / n} T_{2}}{1-e^{-2 k \pi i / n} T_{2}} .
$$

\section{Hirzebruch class of a crepant resolution}

The elliptic genus was defined by many authors. We focus on the version of Borisov and Libgober. In [BL00] a historical account is given and different versions of the elliptic genus are discussed. The elliptic genus generalizes the Hirzebruch class and behaves well with respect to crepant resolutions. First we review basic necessary constructions of Borisov and Libgober [BL03], [BL05] and next we specialize the results of [BL05] to the Hirzebruch class of $\mathbb{C}^{n} / G$. Our goal is to give a formula for the Hirzebruch class of a crepant resolution in terms of Molien series.

\subsection{Elliptic genus}

Let us define the theta function ${ }^{3}$

$$
\begin{aligned}
\theta(v, \tau) & =\frac{1}{i} \sum_{n=-\infty}^{\infty}(-1)^{n} q^{\frac{1}{2}\left(n+\frac{1}{2}\right)^{2}} e^{(2 n+1) \pi i v}= \\
& =2 \sum_{n=0}^{\infty}(-1)^{n} q^{\frac{1}{2}\left(n+\frac{1}{2}\right)^{2}} \sin ((2 n+1) \pi v),
\end{aligned}
$$

\footnotetext{
${ }^{3}$ We give a definition according to [Cha85] but following [BL00], [BL03], [BL05] we set $q=e^{2 \pi i \tau}$ not $q=e^{\pi i \tau}$. Therefore we have to divide the exponents of $q$ by 2 .
} 
where $q=e^{2 \pi i \tau}$, see [Cha85, Chap. V.1]. The series is convergent for $\operatorname{Im}(\tau)>0$ (i.e., $|q|<1$ ) and $v \in \mathbb{C}$, but we treat it only as a power series in $v$ with a parameter $\tau$. According to Jacobi product formula [Cha85, Ch V.6]

$$
\theta(v, \tau)=q^{1 / 8} 2 \sin (\pi v) \prod_{l=1}^{l=\infty}\left(1-q^{l}\right) \prod_{l=1}^{l=\infty}\left(1-q^{l} e^{2 \pi i v}\right)\left(1-q^{l} e^{-2 \pi i v}\right) .
$$

For a smooth complex variety the elliptic class is defined in terms of the Chern roots $x_{i}$ of the tangent bundle as

$$
\mathcal{E} \mathcal{L} \mathcal{L}(Y ; z, \tau)=\prod_{k=1}^{\operatorname{dim}(Y)} x_{k} \frac{\theta\left(x_{k} / 2 \pi i-z, \tau\right)}{\theta\left(x_{k} / 2 \pi i, \tau\right)} \in H^{*}(Y) \otimes \mathbb{C}[[z, \tau]] .
$$

The elliptic genus is the integral

$$
\int_{Y} \mathcal{E} \mathcal{L} \mathcal{L}(Y ; z, \tau)
$$

Let us take the limit of the elliptic class when $\tau \rightarrow i \infty$ (or when $q \rightarrow 0$ ). By the Jacobi product formula (15) we have

$$
\begin{aligned}
\lim _{\tau \rightarrow i \infty} \frac{\theta(x / 2 \pi i-z, \tau)}{\theta(x / 2 \pi i, \tau)} & =\frac{\sin (\pi(x / 2 \pi i-z))}{\sin (\pi x / 2 \pi i)} \\
& =\frac{e^{\pi i(x / 2 \pi i-z)}-e^{-\pi i(x / 2 \pi i-z)}}{e^{\pi i x / 2 \pi i}-e^{-\pi i x / 2 \pi i}}=e^{-\pi i z} \frac{\left(1-e^{2 \pi i z} e^{-x}\right)}{\left(1-e^{-x}\right)} .
\end{aligned}
$$

Therefore

$$
\lim _{\tau \rightarrow i \infty} \mathcal{E} \mathcal{L} \mathcal{L}(Y ; z, \tau)=e^{-\operatorname{dim}(Y) \pi i z} \operatorname{td}_{-e^{2 \pi i z}}(Y),
$$

which can be written as

$$
(-y)^{-\frac{\operatorname{dim}(Y)}{2}} \operatorname{td}_{y}(Y), \quad \text { with } y=-e^{2 \pi i z} .
$$

Note that in [BL00], [BL05] $e^{2 \pi i z}=y$, but we want to have a formula which agrees with our convention for $\chi_{y}$ genus, thus we introduce the minus sign.

Then a relative elliptic genus for Kawamata log-terminal pairs $(Y, D)$ is introduced; for definition see [Bat99, Def. 3.7] or [BL05, §2]. If $f: Y \rightarrow X$ is a resolution of a variety with (at most) $\mathbb{Q}$-Gorenstein singularities and $D=K_{Y}-f^{*} K_{X}$, then the relative elliptic genus is independent of the resolution. This way one obtains an invariant of singular varieties, see [BL03, Prop. 3.6, 3.7]. The construction is local and allows us to define the characteristic class $\mathcal{E} \mathcal{L} \mathcal{L}(X ; z, \tau)$ for varieties with $\mathbb{Q}$-Gorenstein singularities. If $f: Y \rightarrow X$ is a crepant resolution then

$$
\mathcal{E} \mathcal{L} \mathcal{L}(X ; z, \tau)=f_{*}(\mathcal{E} \mathcal{L} \mathcal{L}(Y ; z, \tau)) \in H_{*}(X) \otimes \mathbb{C}[[z, \tau]] .
$$

In particular, $f_{*}(\mathcal{E} \mathcal{L} \mathcal{L}(Y ; z, \tau))$ does not depend on the crepant resolution. 
Corollary 11. Suppose $X$ has at most $\mathbb{Q}$-Gorenstein singularities. For a crepant resolution $f: Y \rightarrow X$ the push-forward

$$
f_{*}\left(\operatorname{td}_{y}(Y)\right) \in H_{*}(X) \otimes \mathbb{C}[y]
$$

does not depend on $Y$.

Note that for symplectic quotients of dimension 4 Corollary 11 follows from [AW14, Thm. 3.2] where it is shown that any two crepant resolutions differ by a sequence of flops.

\subsection{Orbifold elliptic genus}

Suppose a finite group $G$ acts on a complex manifold $Y$. For any two commuting elements $g, h \in G$ denote by $Y^{g, h}$ the fixed point set for both elements. For the sake of simplicity we assume that $Y^{g, h}$ is connected. For a pair of commuting elements $g, h$ we decompose the bundle $\left.T Y\right|_{Y^{g, h}} \simeq \bigoplus_{\lambda} V_{\lambda}$ into common eigen-subbundles. Let $x_{\lambda}$ be the first Chern class of $V_{\lambda}$. (We assume that $\operatorname{dim}\left(V_{\lambda}\right)=1$, otherwise we use the splitting principle.) Suppose that $g$ (resp. $h$ ) acts on $V_{\lambda}$ via multiplication by $e^{2 \pi i \lambda(g)}$ with $\lambda(g) \in \mathbb{Q} \cap[0,1)$ (resp. by $\left.e^{2 \pi i \lambda(h)}, \lambda(h) \in \mathbb{Q} \cap[0,1)\right)$. The orbifold elliptic class is defined by the formula

$$
\begin{aligned}
& \mathcal{E} \mathcal{L} \mathcal{L}_{\mathrm{orb}}(Y, G ; z, \tau) \\
& =\frac{1}{|G|} \sum_{g h=h g}\left(i_{Y^{g}, h}\right)^{*}\left(\prod_{\lambda(g)=\lambda(h)=0} x_{\lambda} \prod_{\lambda} \frac{\theta\left(x_{\lambda} / 2 \pi i+\lambda(g)-\tau \lambda(h)-z, \tau\right)}{\theta\left(x_{\lambda} / 2 \pi i+\lambda(g)-\tau \lambda(h), \tau\right)} e^{2 \pi i \lambda(h) z}\right),
\end{aligned}
$$

where $i_{Y^{g, h}}: Y^{g, h} \rightarrow Y$ is the inclusion. Note ${ }^{4}$ that in the summation the numbers $\lambda(g)$ and $\lambda(h)$ in fact depend on the pair $(g, h)$, because the decomposition of $\mathbb{C}^{n}$ into eigenspaces of $g$ has to be $h$-invariant. The main result of [BL05] is the following

Theorem 12 ([BL05, Thm. 5.3]). Let $X=Y / G$ be a quotient variety with $Y$ smooth. Suppose $\pi^{*}\left(K_{X}\right)=K_{Y}$, where $\pi$ is the quotient map. Then

$$
\pi_{*}\left(\mathcal{E} \mathcal{L} \mathcal{L}_{\text {orb }}(Y, G ; z, \tau)\right)=\mathcal{E} \mathcal{L} \mathcal{L}(X ; z, \tau) \in H_{*}(X) \otimes \mathbb{C}[[z, \tau]]
$$

Therefore if $f: \tilde{X} \rightarrow X$ is a crepant resolution of the quotient variety, then

$$
\pi_{*}\left(\mathcal{E} \mathcal{L} \mathcal{L}_{\text {orb }}(Y, G ; z, \tau)\right)=f_{*}(\mathcal{E} \mathcal{L} \mathcal{L}(\widetilde{X} ; z, \tau))
$$

In fact Borisov and Libgober prove an equality for quotients of $G$-Kawamata log-terminal $G$-normal pairs ([BL05, Def. 3.2]). The second equality given here follows from the birational invariance of the elliptic class ([BL05, Thm. 3.7]).

\footnotetext{
${ }^{4}$ Instead of $\lambda(g)$ we should have written $\lambda_{k}^{g, h}(g)$ with $k=1, \ldots, \operatorname{dim}\left(X^{g, h}\right)$ and $\lambda_{k}^{g, h}(h)$ instead of $\lambda(h)$, but we do not want to make the formula complicated and we keep the notation of [BL05].
} 


\subsection{An equivariant version of the elliptic class}

If a torus $\mathbb{C}^{*}$ (or any other algebraic group) acts on a $G$-variety $Y$ and the actions commute, then one can define equivariant elliptic cohomology classes

$$
\mathcal{E} \mathcal{L} \mathcal{L}^{\mathbb{C}^{*}}(Y, G ; z, \tau) \text { and } \mathcal{E} \mathcal{L} \mathcal{L}_{\text {orb }}^{\mathbb{C}^{*}}(\tilde{Y} ; z, \tau) \text { in } \widehat{H}_{\mathbb{C}^{*}}^{*}(Y) \otimes \mathbb{C}[[z, \tau]]
$$

by applying the formulas (16) and (19) to equivariant tangent bundles. We approximate the Borel construction by $\left(\mathbb{C}^{m+1} \backslash\{0\}\right) \times \mathbb{C}^{*} Y$. The equivariant elliptic class is approximated by

$$
\left(p_{m}\right)^{*} \mathcal{E} \mathcal{L} \mathcal{L}\left(\mathbb{P}^{m} ; z, \tau\right)^{-1} \cap \mathcal{E} \mathcal{L} \mathcal{L}\left(\left(\mathbb{C}^{m+1} \backslash\{0\}\right) \times \mathbb{C}^{*} Y, G ; z, \tau\right),
$$

where $p_{m}:\left(\mathbb{C}^{m+1} \backslash\{0\}\right) \times \mathbb{C}^{*} Y \rightarrow \mathbb{P}^{m}$ is the projection. Theorem 12 is applied to the twisted product $\left.\mathbb{C}^{m+1} \backslash\{0\}\right) \times \mathbb{C}^{*} Y$ and in the limit we obtain the equality for the equivariant classes.

The straight-forward verification of the formula (18) in the equivariant context was done in [Wae08a]. The equivariant counterpart of McKay correspondence for elliptic genus, i.e., the equivariant version of Theorem 12, was proved in [Wae08b]. Recently the equivariant elliptic class in the context of equivariant localization was studied in [Lib15].

\subsection{Comparison with Molien series}

Let $Y=\mathbb{C}^{n}, G \subset \mathrm{GL}_{n}(\mathbb{C})$. The group $\mathbb{C}^{*}$ is acting on $\mathbb{C}^{n}$ via scalar multiplication. We study the equivariant version of the orbifold elliptic class and its limit when $\tau \rightarrow i \infty$. We will show that the limit can be expressed by the extended Molien series of centralizers of elements of $G$. Let us introduce some notation.

For a group $H$ acting on a vector space $W$ let us denote by $\operatorname{Mol}(H, W ; v, T)$ the extended Molien series.

Recall that the age of an element $g \in \mathrm{GL}_{n}(\mathbb{C})$ of finite order is defined as $\sum_{k=1}^{n} \lambda_{k}$, where $e^{2 \pi i \lambda_{k}}, k=1, \ldots, n$ are the eigenvalues of $g$ and $\lambda_{k} \in[0,1) \cap \mathbb{Q}$.

We will prove that

Theorem 13. Let $G \subset \mathrm{GL}_{n}(\mathbb{C})$. Then

$$
\lim _{\tau \rightarrow i \infty} \mathcal{E} \mathcal{L} \mathcal{L}_{\text {orb }}^{\mathbb{C}^{*}}\left(\mathbb{C}^{n}, G ; z, \tau\right)=t^{n}(-y)^{-n / 2} \sum_{h \in \operatorname{Conj}(G)}(-y)^{\operatorname{age}(h)} \operatorname{Mol}\left(C(h),\left(\mathbb{C}^{n}\right)^{h} ; y T, T\right),
$$

where $T=e^{-t}$.

If $G \subset \mathrm{SL}_{n}(\mathbb{C})$, then age $(g)$ is an integer and

Corollary 14. Let $G \subset \mathrm{SL}_{n}(\mathbb{C})$ and let $f: \tilde{X} \rightarrow X=\mathbb{C}^{n} / G \hookrightarrow M$ be a crepant resolution. Then

$$
\frac{f_{*} \operatorname{td}_{y}^{\mathbb{C}^{*}}(\tilde{X})}{\operatorname{eu}(M, 0)}=\sum_{h \in \operatorname{Conj}(G)}(-y)^{\operatorname{age}(h)} \operatorname{Mol}\left(C(h),\left(\mathbb{C}^{n}\right)^{h} ; y T, T\right),
$$

where $C(h)$ is the centralizer of $h$ in $G$ and $M$ is an ambient space containing $\mathbb{C}^{n} / G$. 
Proof. Having in mind that each $x_{\lambda}=t$ and that the action of $\left(i_{g, h}\right)_{*}$ is the multiplication by $t^{\operatorname{codim}\left(Y^{g, h}\right)}$ we rewrite the definition of the elliptic class

$$
\begin{aligned}
\mathcal{E} \mathcal{L}_{\mathcal{L}_{\text {orb }} \mathbb{C}^{*}}\left(\mathbb{C}^{n}, G ; z, \tau\right) & \\
= & \frac{1}{|G|} \sum_{h \in G} \sum_{g \in C(g)} t^{n} \prod_{\lambda} \frac{\theta(t / 2 \pi i+\lambda(g)-\tau \lambda(h)-z, \tau)}{\theta(t / 2 \pi i+\lambda(g)-\tau \lambda(h), \tau)} e^{2 \pi i \lambda(h) z} .
\end{aligned}
$$

Let us study the limit of the class $\left.\mathcal{E} \mathcal{L} \mathcal{L}_{\text {orb }}^{\mathbb{C}^{*}}\left(\mathbb{C}^{n}, G ; z, \tau\right)\right|_{0}$ when $\tau \rightarrow i \infty$. First observe that

$$
\begin{aligned}
\lim _{\tau \rightarrow i \infty} \frac{\theta(a-\lambda \tau-z)}{\theta(a-\lambda \tau)} & =\lim _{\tau \rightarrow i \infty} \frac{\sin (\pi(a-\lambda \tau-z))}{\sin (\pi(a-\lambda \tau))} \\
& =\lim _{s \rightarrow \infty} \frac{e^{\pi i(a-\lambda i s-z)}-e^{-\pi i(a-\lambda i s-z)}}{e^{\pi i(a-\lambda i s)}-e^{-\pi i(a-\lambda i s)}} \\
& =\lim _{s \rightarrow \infty} \frac{e^{\pi(\lambda s+i(a-z))}-e^{-\pi(\lambda s+i(a-z))}}{e^{\pi(\lambda s+i a)}-e^{-\pi(\lambda s+i a)}}=e^{-\pi i z}
\end{aligned}
$$

for $\lambda \in(0,1)$. Also, we will apply the equality (17) with $x / 2 \pi i+\lambda(g)$ instead of $x / 2 \pi i$. Therefore

$$
\begin{aligned}
\lim _{\tau \rightarrow \infty} & \mathcal{E} \mathcal{L L}_{\text {orb }}^{\mathbb{C}^{*}}(Y, G ; z, \tau) \\
& =\frac{t^{n}}{|G|} \sum_{h \in G} \sum_{g \in C(g)} \prod_{\lambda: \lambda(h)>0} e^{-\pi i z} e^{2 \pi i \lambda(h) z} \prod_{\lambda: \lambda(h)=0} e^{-\pi i z} \frac{1-e^{2 \pi i z} e^{-(t+2 \pi i \lambda(g))}}{1-e^{-(t+2 \pi i \lambda(g))}} e^{2 \pi i \lambda(h) z} .
\end{aligned}
$$

Now setting $T=e^{-t}, e^{2 \pi i z}=-y$, with the convention that $(-y)^{1 / 2}=e^{\pi i z}$ we obtain

$$
\begin{aligned}
\frac{t^{n}}{|G|} \sum_{h \in G} \sum_{g \in C(g)} e^{-n \pi i z} e^{2 \pi i \sum_{\lambda} \lambda(h) z} \prod_{\lambda: \lambda(h)=0} \frac{1-e^{2 \pi i z} e^{-(t+2 \pi i \lambda(g))}}{1-e^{-(t+2 \pi i \lambda(g))}} \\
=\frac{t^{n}}{|G|} \sum_{h \in G} \sum_{g \in C(g)}(-y)^{-\frac{n}{2}}(-y)^{\operatorname{age}(h)} \prod_{\lambda: \lambda(h)=0} \frac{1-y a_{\lambda}(g)^{-1} T}{1-a_{\lambda}(g)^{-1} T} .
\end{aligned}
$$

Here $a_{\lambda}(g)=e^{2 \pi i \lambda(g)}$ is an eigenvalue of $g$. Finally we obtain

$$
\begin{aligned}
& \frac{t^{n}(-y)^{-n / 2}}{|G|} \sum_{h \in G} \sum_{g \in C(g)}(-y)^{\operatorname{age}(h)} \frac{\operatorname{det}\left(\operatorname{Id}+\left.y T g\right|_{\left.\left(\mathbb{C}^{n}\right)^{h}\right)}\right.}{\operatorname{det}\left(\operatorname{Id}-\left.T g\right|_{\left(\mathbb{C}^{n}\right)^{h}}\right)} \\
& =t^{n}(-y)^{-\frac{n}{2}} \sum_{[h] \in \operatorname{Conj}(G)} \frac{1}{|C(g)|} \sum_{g \in C(g)}(-y)^{\operatorname{age}(h)} \frac{\operatorname{det}\left(I d+\left.y T g\right|_{\left.\left(\mathbb{C}^{n}\right)^{h}\right)}\right.}{\operatorname{det}\left(\operatorname{Id}-\left.T g\right|_{\left.\left(\mathbb{C}^{n}\right)^{h}\right)}\right.} \\
& =t^{n}(-y)^{-n / 2} \sum_{[h] \in \operatorname{Conj}(G)}(-y)^{\operatorname{age}(h)} \operatorname{Mol}\left(C(h),\left(\mathbb{C}^{n}\right)^{h} ; y T, T\right) . \quad \square
\end{aligned}
$$


Theorem 13 can be interpreted as

$$
\frac{1}{\mathrm{eu}(M, 0)} f_{*} \operatorname{td}_{y}^{\mathbb{C}^{*}}(\widetilde{X})=\frac{1}{\mathrm{eu}(M, 0)} f_{*} \operatorname{td}_{y}^{\mathbb{C}^{*}}\left(\widehat{\mathbb{C}^{n} / G}\right),
$$

where

$$
\widehat{\mathbb{C}^{n} / G}=\bigsqcup_{h \in \operatorname{Conj}(G)}\left(\mathbb{C}^{n}\right)^{h} / C(h) \times \mathbb{C}^{\operatorname{age}(h)} .
$$

This space only slightly differs from the so-called inertia stack

$$
\bigsqcup_{h \in \operatorname{Conj}(G)}\left(\mathbb{C}^{n}\right)^{h} / C(h)=\left\{(x, g) \in \mathbb{C}^{n} \times G \mid g x=x\right\} / G,
$$

defined already in [BC88].

\subsection{Divisibility}

Denote by $\widetilde{H}(y, T)$ the sum

$$
\sum_{[h] \in \operatorname{Conj}(G)}(-y)^{\operatorname{age}(h)} \operatorname{Mol}\left(C(h),\left(\mathbb{C}^{n}\right)^{h} ; y T, T\right) .
$$

By Theorem 13

$$
\frac{f_{*} \operatorname{td}_{y}^{\mathbb{C}^{*}}(\widetilde{X})}{\operatorname{eu}(M, 0)}=\widetilde{H}(y, T)
$$

for a crepant resolution of a quotient variety $\mathbb{C}^{n} / G$. By [Web16b]

$$
\lim _{T \rightarrow 0} \frac{f_{*} \operatorname{td}_{y}^{\mathbb{C}^{*}}(\widetilde{X})}{\operatorname{eu}(M, 0)}=\chi_{y}\left(f^{-1}(0)\right) .
$$

If $f$ is crepant, then this limit is equal to $\widetilde{H}(y, 0)=\sum_{g \in \operatorname{Conj}(G)}(-y)^{\operatorname{age}(g)}$. By [Bat99, Thm. 8.4] the cohomology of $f^{-1}(0)$ is pure of Hodge type $(k, k)$. It follows that

$$
\widetilde{H}(-x, 0)=\sum b_{2 k}\left(f^{-1}(0)\right) x^{k} \quad \text { and } \quad \widetilde{H}(-1,0)=\chi_{\text {top }}\left(f^{-1}(0)\right)=|\operatorname{Conj}(G)| .
$$

Proposition 15. If $G \subset \mathrm{Sp}_{n}(\mathbb{C})$, then

$$
\widetilde{H}(y, T)-(-y)^{n} \widetilde{H}(-1,0)
$$

is divisible by $(y+1)\left(1+T^{2} y\right)$.

Proof. By Proposition 7

$$
(-y)^{\operatorname{age}(h)} \operatorname{Mol}\left(C(h),\left(\mathbb{C}^{2 n}\right)^{h}, v T, T\right)-(-y)^{\operatorname{age}(h)+\frac{1}{2} \operatorname{dim}\left(\left(\mathbb{C}^{2 n}\right)^{h}\right.}
$$

is divisible by $(1+y)\left(1+T^{2} y\right)$. For symplectic actions age $(h)=\operatorname{codim}\left(\left(\mathbb{C}^{2 n}\right)^{h}\right) / 2$, see [Kal02]. The exponent of $(-y)$ is equal to

$$
\frac{\operatorname{codim}\left(\left(\mathbb{C}^{2 n}\right)^{h}\right.}{2}+\frac{\operatorname{dim}\left(\left(\mathbb{C}^{2 n}\right)^{h}\right.}{2}=n
$$

To have divisibility of $\widetilde{H}(y, T)$ we have to subtract $(-y)^{n}$ for each summand of (20), i.e., for each conjugacy class $[h] \in \operatorname{Conj}(G)$. 


\section{Final remarks, positivity}

The initial work of the second author [Web16a] was directed towards the search of positivity results. We can check that in our examples (and in many others) after the substitution $T:=1+S$ and $y:=-1-\delta$ the numerator of $H(y, T)$ is a polynomial with nonnegative coefficients. That is always the case for simplicial toric varieties by [Web16a, Thm. 13.1]. The proof is based on the formula for the Hirzebruch class of a toric variety, Proposition 9. Any representation of an abelian finite group can be diagonalized, therefore the quotient variety admits an action of the full torus. Hence such quotient is a simplicial toric variety and the positivity holds. For general quotient varieties we have no proof, except from a partial result, Proposition 16 and Corollary 17.

Proposition 16. If $G \subset \mathrm{Sp}_{2 n}(\mathbb{C})$ then after the substitution

$$
T:=1+S \quad \text { and } \quad y:=-1-\delta
$$

the Hirzebruch class $H(y, T)$ for $\mathbb{C}^{n} / G$ can be written as a quotient of polynomials with nonnegative coefficients. The polynomial in the denominator has roots in the unit circle.

Proof. We look at component of $H(y, T)$ corresponding to $g \in G$. If $\varepsilon$ is an eigenvalue of a matrix $g \in G$ then, since $g$ is symplectic, $\bar{\varepsilon}=\varepsilon^{-1}$ is also an eigenvalue of $g$. Moreover, eigenvalues 1 and -1 appear with even multiplicities. Thus we may write

$$
\begin{aligned}
\frac{P_{g}(y, T)}{Q_{g}(T)} & =\prod_{k=1}^{2 n} \frac{1+a_{k}(g) y T}{1-a_{k}(g) T} \\
& =\frac{\left(\prod_{k=1}^{n_{1}}\left(1+a_{k}(g) y T\right)\left(1+\overline{a_{k}(g)} y T\right)\right)(1+y T)^{2 n_{2}}(1-y T)^{2 n_{3}}}{\left(\prod_{k=1}^{n_{1}}\left(1-a_{k}(g) T\right)\left(1-\overline{a_{k}(g)} T\right)\right)(1+T)^{2 n_{2}}(1-T)^{2 n_{3}}} .
\end{aligned}
$$

After given substitutions $(1-y T)^{2},(1+y T)^{2},(1+T)^{2},(1-T)^{2}$ have nonnegative coefficients as polynomials in $\delta, S$. Also, for $\varepsilon$ from the unit circle and a polynomial $P$ we have

$$
\begin{aligned}
(1-\varepsilon(1+P))(1-\bar{\varepsilon}(1+P)) & =1-(\varepsilon+\bar{\varepsilon})(1+P)+(1+P)^{2} \\
& =2+2 P-(\varepsilon+\bar{\varepsilon})(1+P)+P^{2} \\
& =(2-\varepsilon-\bar{\varepsilon})(1+P)+P^{2},
\end{aligned}
$$

which has nonnegative coefficients if $P$ has. This shows that for any eigenvalue $a_{k}(g)$ both $\left(1+a_{k}(g) y T\right)\left(1+\overline{a_{k}(g)} y T\right)$ and $\left(1-a_{k}(g) T\right)\left(1-\overline{a_{k}(g)} T\right)$ have nonnegative coefficients in $\delta, S$.

Thus by formula (2) and Theorem 1 we see that

$$
H(y, T)=\sum_{g \in G} \frac{P_{g}(y, T)}{Q_{g}(T)}
$$


is a sum of fractions where $P_{g}$ and $Q_{g}$ are products of indecomposable (over $\mathbb{R}$ ) factors; each factor has nonnegative coefficients after the considered substitutions. When we reduce the sum of fractions to the common denominator, then both numerator and denominator will have nonnegative coefficients after the substitutions.

Multiplying the numerator and the denominator by $(1-\varepsilon T)(1-\bar{\varepsilon} T)$, where $\varepsilon$ is a root of unity, we can achieve the product of the factors $1-T^{k_{i}}$ in the denominator, not losing the positivity of the numerator.

Corollary 17. If $G \subset \mathrm{Sp}_{2 n}(\mathbb{C})$ then the Hirzebruch class for $\mathbb{C}^{n} / G$ can be written as

$$
H(y, T)=\frac{P(y, T)}{\prod_{i=1}^{r}\left(1-T^{k_{i}}\right)},
$$

where $P(-1-\delta, 1+S)$ is a polynomial in $S$ and $\delta$ with nonnegative coefficients.

The equivariant Hirzebruch class specializes to the Chern-Schwartz-MacPherson class (see [BSY10, §1]). For an equivariant embedding $i: X \hookrightarrow M$ the image $i_{*}\left(c_{S M}^{\mathbb{C}^{*}}\right)(X)$ is a polynomial in $t \in H^{2} \mathbb{C}^{*}(p t)$ of degree $\operatorname{dim}(M)$ and divided by $\mathrm{eu}(M, 0)$ does not depend on $M$. We have

$$
\lim _{\delta \rightarrow 0} H\left(-1-\delta, e^{\delta t}\right)=\frac{i_{*}\left(c_{S M}^{\mathbb{C}^{*}}\right)(X)}{\mathrm{eu}(M, 0)} .
$$

Proposition 18. If $G \subset \mathrm{GL}_{n}(\mathbb{C})$ then the equivariant Chern-Schwartz-MacPherson class is equal to

$$
i_{*}\left(c_{S M}^{\mathbb{C}^{*}}\right)(X)=\frac{\mathrm{eu}(M, 0)}{|G|} \sum_{g \in G}\left(\frac{1+t}{t}\right)^{\operatorname{dim}\left(\left(\mathbb{C}^{n}\right)^{g}\right)} \in H_{\mathbb{C}^{*}}^{*}(M) \simeq \mathbb{Q}[t] .
$$

Proof. First note that

$$
\lim _{\delta \rightarrow 0} \frac{1-a(1+\delta) e^{\delta t}}{1-a e^{\delta t}}=\left\{\begin{array}{cl}
(1+t) / t & \text { for } a=1 \\
1 & \text { for } a \neq 1
\end{array}\right.
$$

To compute the limit (21) we apply formula (2) and Theorem 1

$$
\begin{aligned}
\frac{i_{*}\left(c_{S M}^{\mathbb{C}_{S}^{*}}\right)(X)}{\mathrm{eu}(M, 0)} & =\lim _{\delta \rightarrow 0} H\left(-1-\delta, e^{\delta t}\right)=\frac{1}{|G|} \sum_{g \in G} \lim _{\delta \rightarrow 0} \frac{\operatorname{det}\left(I-(1+\delta) e^{\delta t} g\right)}{\operatorname{det}\left(I-e^{\delta t} g\right)} \\
& =\frac{1}{|G|} \sum_{g \in G} \prod_{k=1}^{n} \lim _{\delta \rightarrow 0} \frac{1-a_{k}(g)(1+\delta) e^{\delta t}}{1-a_{k}(g) e^{\delta t}} \\
& =\frac{1}{|G|} \sum_{g \in G}\left(\frac{1+t}{t}\right)^{\operatorname{dim}\left(\left(\mathbb{C}^{n}\right)^{g}\right)} \cdot
\end{aligned}
$$


Corollary 19. If $G \subset \mathrm{GL}_{n}(\mathbb{C})$ then the equivariant Chern-Schwartz-MacPherson class

$$
i_{*}\left(c_{S M}^{\mathbb{C}^{*}}\right)(X) \in H_{\mathbb{C}^{*}}^{*}(M) \simeq \mathbb{Q}[t]
$$

for $X=\mathbb{C}^{n} / G$ is a polynomial in $t$ with nonnegative coefficients.

This is a local version of a positivity property, which was studied for Schubert varieties in [AM09] and for hyperplane arrangements [Alu13, §6].

Remark 20. The formulas (7) and (22) imply the analogous statement for ChernSchwartz-MacPherson classes of the global quotient $X=Y / G$, where $Y$ is a smooth variety, and $G$ is a finite group of automorphisms, see [CMSS12, formula $(13)]$ :

$$
c_{S M}(X)=\frac{1}{|G|} \sum_{g \in G}\left(\pi_{g}\right)_{*}\left(c_{S M}\left(Y^{g}\right)\right) .
$$

Similar formulas hold for the equivariant global case. By Theorem 13 for crepant resolutions $f: \widetilde{X} \rightarrow X$

$$
\begin{aligned}
f_{*} c_{S M}(\tilde{X}) & =\sum_{[h] \in \operatorname{Conj}(G)} c_{S M}\left(Y^{h} / C(h)\right) \\
& =\sum_{[h] \in \operatorname{Conj}(G)} \frac{1}{|C[h]|} \sum_{g \in C(h)}\left(\pi_{g, h}\right)_{*}\left(c_{S M}\left(Y^{g, h}\right)\right) \\
& =\frac{1}{|G|} \sum_{g h=h g}\left(\pi_{g, h}\right)_{*}\left(c_{S M}\left(Y^{g, h}\right)\right) .
\end{aligned}
$$

Here $\pi_{g, h}: Y^{g, h} \rightarrow Y / G$ is the projection restricted to the fixed point set $Y^{g, h}$.

The starting point of our common research was to study the Hirzebruch class from the point of view of the existence of symplectic resolution. We have observed certain regularities, especially for $\widetilde{H}(y, T)$ of quotients having a crepant resolution. However, it is hard to grasp a general pattern. We hope it is possible to find a necessary criterion for existence of a crepant resolution. This problem might be an interesting subject of further research.

\section{Appendix: more examples}

If $G \subset \operatorname{Sp}_{n}(\mathbb{C})$ then the crepant resolution of $\mathbb{C}^{n} / G$ is the same as the symplectic resolution, see [Ver00]. All the examples given below are quotients by symplectic groups, and all these groups except example (9) are generated by symplectic reflections (i.e., matrices such that the eigenspace for the eigenvalue 1 is of dimension $n-2)$. Again by [Ver00], this last condition is a necessary one for the existence of a symplectic resolution. Note that all irreducible matrix groups generated by symplectic reflections can be found in Cohen's classification [Coh80].

For the sake of simplicity most of our examples are 4-dimensional. We give $H(y, T)=\operatorname{Mol}(y T, T)$ and $\widetilde{H}(y, T)$ in the simplified fraction form. The computations presented here were performed in the hope of discovering whether the form of the equivariant Hirzebruch class is related to certain properties of the given quotient or its resolutions. 


\subsection{List of tested groups}

(1) Du Val singularities.

(2) A 4-dimensional symplectic group with 32 elements, which is isomorphic to $Q_{8} \times_{\mathbb{Z}_{2}} D_{8}$, where $Q_{8}$ is the quaternion group and $D_{8}$ is the dihedral group of 8 elements. The group is the first element of the second infinite series in the first part of Cohen's classification [Coh80, Table I]. By [BS13] symplectic resolutions of the corresponding quotient singularity exist; they were constructed in [DBW14].

(3) A reducible 4-dimensional symplectic representation of the binary tetrahedral group. It is obtained from a 2-dimensional representation by taking a direct sum with its contragradient representation. There are 2 symplectic resolutions for the corresponding quotient singularity; they were constructed in [LS12].

(4) A 4-dimensional symplectic representation of the dihedral group D8, constructed as a wreath product $\mathbb{Z}_{2} \prec S_{2}$. This is an element of an infinite series of (reducible) symplectic representations, for which a symplectic resolution can be constructed using a suitable Hilbert scheme.

(5) A 4-dimensional symplectic representation of the symmetric group $S_{3}$. This is the only 4-dimensional element of another infinite series of symplectic representations (of symmetric groups $S_{n}$ ) for which symplectic resolutions come from a Hilbert scheme construction.

(6) A 4-dimensional symplectic group of order 16 , a semidirect product $\left(\mathbb{Z}_{4} \times\right.$ $\left.\mathbb{Z}_{2}\right) \rtimes \mathbb{Z}_{2}$. It appears for $m=2$ in the 7 th infinite series in [Coh80, Table I]. It is a subgroup of the 32-element group (2). It is not known whether a symplectic resolution exists.

(7) A 4-dimensional symplectic group of order 24 , a semidirect product $\left(\mathbb{Z}_{6} \times\right.$ $\left.\mathbb{Z}_{2}\right) \rtimes \mathbb{Z}_{2}$. It appears for $m=3$ in the 7 th series in [Coh80, Table I]. It is not known whether a symplectic resolution exists.

(8) A 4-dimensional group of order 64 , isomorphic to $\left(\mathbb{Z}_{4} \rtimes Q_{8}\right) \rtimes \mathbb{Z}_{2}$. It appears in the 1st series (for $m=2$ ) in [Coh80, Table I]; in particular, it is generated by symplectic reflections. However, by [BS13] symplectic resolutions do not exist.

(9) The smallest (imprimitive) group generated by symplectic reflection in dimension 6 . It is a representation of the symmetric group $S_{4}$, constructed from $\mathbb{Z}_{2}$ and the trivial group as described in [Coh80, Not. 2.8]. The symplectic resolution does not exist by [BS13, Thm. 7.2].

(10) A representation of $\mathbb{Z}_{5}$ without symplectic reflections. The considered subgroup $\mathbb{Z}_{5} \subset \mathrm{SL}_{4}(\mathbb{C})$ is generated by $\operatorname{diag}\left(\xi^{1}, \xi^{2}, \xi^{3}, \xi^{4}\right)$. Note that this is not an action by symplectic reflections and therefore there is no symplectic, i.e., crepant, resolution.

\subsection{Results for $H(y, T)$ and $\widetilde{H}(y, T)$}

(1) The Molien series of Du Val singularities are given in $§ 5.2$. The Molien series of the crepant resolutions are obtained by subtracting $k y$, where $k$ is the number of the components of the exceptional divisor. 
(2) $Q_{8} \times_{\mathbb{Z}_{2}} D_{8}$, order 32, dimension 4 .

$$
\begin{aligned}
H(y, T)= & y^{2}+\frac{(y+1)\left(T^{2} y+1\right)}{\left(1-T^{2}\right)^{2}\left(1-T^{4}\right)^{2}} \cdot\left(\left(T^{8}-2 T^{6}+4 T^{4}-2 T^{2}+1\right)\left(T^{2} y^{2}+1\right)\right. \\
& \left.+\left(-T^{10}+2 T^{8}+T^{6}+T^{4}+2 T^{2}-1\right) y\right) \\
\widetilde{H}(y, T)= & 17 y^{2}+\frac{(1+y)\left(1+T^{2} y\right)}{\left(1-T^{2}\right)^{2}\left(1-T^{4}\right)^{2}} \cdot\left(\left(1-2 T^{2}+4 T^{4}-2 T^{6}+T^{8}\right)\left(T^{2} y^{2}+1\right)\right. \\
& \left.-2\left(T^{2}+1\right)\left(3 T^{8}-9 T^{6}+11 T^{4}-9 T^{2}+3\right) y\right)
\end{aligned}
$$

(3) Binary tetrahedral group, dimension 4.

$$
\begin{aligned}
H(y, T)= & y^{2}+\frac{(y+1)\left(T^{2} y+1\right)}{\left(1-T^{2}\right)\left(1-T^{4}\right)^{2}\left(1-T^{6}\right)} \cdot\left(\left(T^{12}+2 T^{8}+2 T^{6}+2 T^{4}+1\right)\right. \\
& \left.\cdot\left(T^{2} y^{2}+1\right)+\left(-T^{14}+T^{12}+4 T^{10}+4 T^{8}+4 T^{6}+4 T^{4}+T^{2}-1\right) y\right) \\
\widetilde{H}(y, T)= & 7 y^{2}+\frac{(y+1)\left(T^{2} y+1\right)}{\left(1-T^{2}\right)\left(1-T^{4}\right)^{2}\left(1-T^{6}\right)} \cdot\left(\left(T^{12}+2 T^{8}+2 T^{6}+2 T^{4}+1\right)\right. \\
& \left.\cdot\left(T^{2} y^{2}+1\right)-\left(T^{2}+1\right)\left(3 T^{12}-4 T^{8}-6 T^{6}-4 T^{4}+3\right) y\right)
\end{aligned}
$$

(4) Dihedral group $D_{8}$, dimension 4 .

$$
\begin{aligned}
H(y, T)= & y^{2}+\frac{(y+1)\left(T^{2} y+1\right)}{\left(1-T^{2}\right)^{2}\left(1-T^{4}\right)^{2}} \cdot\left(\left(T^{8}+T^{6}+4 T^{4}+T^{2}+1\right)\left(T^{2} y^{2}+1\right)\right. \\
& \left.+\left(-T^{10}+2 T^{8}+7 T^{6}+7 T^{4}+2 T^{2}-1\right) y\right) \\
\widetilde{H}(y, T)= & 5 y^{2}+\frac{(y+1)\left(T^{2} y+1\right)}{\left(1-T^{2}\right)^{2}\left(1-T^{4}\right)^{2}} \cdot\left(\left(T^{8}+T^{6}+4 T^{4}+T^{2}+1\right)\left(T^{2} y^{2}+1\right)\right. \\
& \left.-\left(T^{2}+1\right)\left(3 T^{8}-3 T^{6}-8 T^{4}-3 T^{2}+3\right) y\right)
\end{aligned}
$$

(5) Symmetric group $S 3$, dimension 4 .

$$
\begin{gathered}
H(y, T)=y^{2}+\frac{(y+1)\left(T^{2} y+1\right)}{\left(1-T^{2}\right)^{2}\left(1-T^{3}\right)^{2}} \cdot\left(\left(T^{6}+T^{4}+2 T^{3}+T^{2}+1\right)\left(T^{2} y^{2}+1\right)\right. \\
\left.+\left(-T^{8}+2 T^{6}+4 T^{5}+2 T^{4}+4 T^{3}+2 T^{2}-1\right) y\right) \\
\widetilde{H}(y, T)=3 y^{2}+\frac{(y+1)\left(T^{2} y+1\right)}{\left(1-T^{2}\right)^{2}\left(1-T^{3}\right)^{2}} \cdot\left(\left(T^{6}+T^{4}\right.\right. \\
\left.+2 T^{3}+T^{2}+1\right)\left(T^{2} y^{2}+1\right) \\
\left.-\left(T^{2}+1\right)\left(2 T^{6}+2 T^{5}-3 T^{4}-8 T^{3}-3 T^{2}+2 T+2\right) y\right)
\end{gathered}
$$

(6) $\left(\mathbb{Z}_{4} \times \mathbb{Z}_{2}\right) \rtimes \mathbb{Z}_{2}$, order 16 , dimension 4 . 


$$
\begin{aligned}
H(y, T)= & y^{2}+\frac{(y+1)\left(T^{2} y+1\right)}{\left(1-T^{2}\right)^{2}\left(1-T^{4}\right)^{2}} \cdot\left(\left(T^{8}-T^{6}\right.\right. \\
& \left.+4 T^{4}-T^{2}+1\right)\left(T^{2} y^{2}+1\right) \\
& \left.+\left(-T^{10}+2 T^{8}+3 T^{6}+3 T^{4}+2 T^{2}-1\right) y\right) \\
\widetilde{H}(y, T)= & 10 y^{2}+\frac{(y+1)\left(T^{2} y+1\right)}{\left(1-T^{2}\right)^{2}\left(1-T^{4}\right)^{2}} \cdot\left(\left(T^{8}-T^{6}\right.\right. \\
& \left.+4 T^{4}-T^{2}+1\right)\left(T^{2} y^{2}+1\right) \\
& \left.-\left(T^{2}+1\right)\left(4 T^{8}-9 T^{6}+6 T^{4}-9 T^{2}+4\right) y\right)
\end{aligned}
$$

(7) $\left(\mathbb{Z}_{6} \times \mathbb{Z}_{2}\right) \rtimes \mathbb{Z}_{2}$, order 24 , dimension 4 .

$$
\begin{aligned}
H(y, T)= & y^{2}+\frac{(y+1)\left(T^{2} y+1\right)}{\left(1-T^{4}\right)^{2}\left(1-T^{6}\right)^{2}} \\
& \cdot\left(T^{16}+T^{14}+2 T^{12}+4 T^{10}+8 T^{8}+4 T^{6}\right. \\
& \left.+2 T^{4}+T^{2}+1\right)\left(T^{2} y^{2}+1\right) \\
& \left.+\left(-T^{18}+3 T^{14}+8 T^{12}+14 T^{10}+14 T^{8}+8 T^{6}+3 T^{4}-1\right) y\right) \\
\widetilde{H}(y, T)=9 y^{2}+\frac{(y+1)\left(T^{2} y+1\right)}{\left(1-T^{4}\right)^{2}\left(1-T^{6}\right)^{2}} & \left.\quad+2 T^{4}+T^{2}+1\right)\left(T^{2} y^{2}+1\right) \\
& \left(\left(T^{16}+T^{14}+2 T^{12}+4 T^{10}+8 T^{8}+4 T^{6}\right.\right. \\
& -\left(T^{2}+1\right)\left(3 T^{16}+T^{14}-2 T^{12}-8 T^{10}\right. \\
- & \left.\left.12 T^{8}-8 T^{6}-2 T^{4}+T^{2}+3\right) y\right)
\end{aligned}
$$

(8) $\left(\mathbb{Z}_{4} \rtimes Q_{8}\right) \rtimes \mathbb{Z}_{2}$, order 64 , dimension 4 .

$$
\begin{aligned}
H(y, T)= & y^{2}+\frac{(y+1)\left(T^{2} y+1\right)}{\left(1-T^{2}\right)^{2}\left(1-T^{4}\right)\left(1-T^{8}\right)} \\
& \cdot\left(\left(T^{12}-2 T^{10}+3 T^{8}-2 T^{6}+3 T^{4}-2 T^{2}+1\right)\left(T^{2} y^{2}+1\right)\right. \\
& \left.\quad+\left(-T^{14}+2 T^{12}+T^{8}+T^{6}+2 T^{2}-1\right) y\right) \\
\widetilde{H}(y, T)= & 16 y^{2}+\frac{(y+1)\left(T^{2} y+1\right)}{\left(1-T^{2}\right)^{2}\left(1-T^{4}\right)\left(1-T^{8}\right)} \\
& \cdot\left(\left(T^{12}-2 T^{10}+3 T^{8}-2 T^{6}+3 T^{4}-2 T^{2}+1\right)\left(T^{2} y^{2}+1\right)\right. \\
& \left.\quad-\left(T^{2}+1\right)\left(5 T^{12}-14 T^{10}+21 T^{8}-26 T^{6}+21 T^{4}-14 T^{2}+5\right) y\right)
\end{aligned}
$$

(9) Symmetric group $S_{4}$, dimension 6 . 


$$
\begin{aligned}
& H(y, T)=-y^{3}+\frac{(y+1)\left(T^{2} y+1\right)}{\left(T^{4}-1\right)^{2}\left(T^{3}-1\right)^{2}\left(T^{2}-1\right)^{2}} \\
& \cdot\left(\left(T^{12}+T^{10}+2 T^{9}+4 T^{8}+2 T^{7}+4 T^{6}\right.\right. \\
& \left.+2 T^{5}+4 T^{4}+2 T^{3}+T^{2}+1\right)\left(y^{4} T^{4}+1\right) \\
& +\left(-T^{14}+2 T^{12}+4 T^{11}+7 T^{10}+10 T^{9}+16 T^{8}+20 T^{7}+16 T^{6}\right. \\
& \left.+10 T^{5}+7 T^{4}+4 T^{3}+2 T^{2}-1\right)\left(y^{2} T^{2}+1\right) y \\
& +\left(T^{16}-T^{14}-2 T^{13}+12 T^{11}+19 T^{10}+30 T^{9}+26 T^{8}\right. \\
& \left.\left.+30 T^{7}+19 T^{6}+12 T^{5}-2 T^{3}-T^{2}+1\right) y^{2}\right) \\
& \widetilde{H}(y, T)=-5 y^{3}+\frac{(y+1)\left(T^{2} y+1\right)}{\left(T^{4}-1\right)^{2}\left(T^{3}-1\right)^{2}\left(T^{2}-1\right)^{2}} \\
& \cdot\left(\left(T^{12}+T^{10}+2 T^{9}+4 T^{8}+2 T^{7}+4 T^{6}+2 T^{5}\right.\right. \\
& \left.+4 T^{4}+2 T^{3}+T^{2}+1\right)\left(T^{4} y^{4}+1\right) \\
& -(T+1)^{2}\left(2 T^{12}-2 T^{11}+4 T^{10}-6 T^{9}+3 T^{8}-12 T^{7}\right. \\
& \left.-2 T^{6}-12 T^{5}+3 T^{4}-6 T^{3}+4 T^{2}-2 T+2\right)\left(T^{2} y^{2}+1\right) y \\
& +2\left(T^{2}+T+1\right)\left(2 T^{14}-T^{13}-2 T^{12}-5 T^{11}-4 T^{10}+6 T^{9}+8 T^{8}\right. \\
& \left.\left.+16 T^{7}+8 T^{6}+6 T^{5}-4 T^{4}-5 T^{3}-2 T^{2}-T+2\right) y^{2}\right)
\end{aligned}
$$

(10) $\mathbb{Z}_{5}$, no symplectic reflections, dimension 4 .

$$
\begin{aligned}
H(y, T)= & y^{2}+\frac{(y+1)\left(y T^{2}+1\right)}{(1-T)^{3}\left(1-T^{5}\right)} \cdot\left(\left(y^{2} T^{2}+1\right)\left(1-3 T+5 T^{2}-3 T^{3}+T^{4}\right)\right. \\
& \left.-\left(1-3 T+2 T^{2}-2 T^{3}+2 T^{4}-3 T^{5}+T^{6}\right) y\right) \\
\widetilde{H}(y, T)= & 5 y^{2}+\frac{(y+1)\left(y T^{2}+1\right)}{(1-T)^{3}\left(1-T^{5}\right)} \cdot\left(\left(T^{4}-3 T^{3}+5 T^{2}-3 T+1\right)\left(T^{2} y^{2}+1\right)\right. \\
& \left.-\left(T^{6}-3 T^{5}+2 T^{4}-2 T^{3}+2 T^{2}-3 T+1\right) y\right)
\end{aligned}
$$

\section{Appendix: Extended Molien series}

We prove formula (2).

For a vector space $V$ denote by $A(V)$ the bigraded vector space

$$
\sum_{k=0}^{\infty} \sum_{\ell=0}^{\operatorname{dim}(V)} \operatorname{Sym}^{k}(V) \otimes \Lambda^{\ell} V .
$$

We have

$$
A(V \oplus W)=A(V) \otimes A(W) .
$$

Let $g: V \rightarrow V$ be a linear map. Let $\tilde{g}: A(V) \rightarrow A(V)$ be the induced map. Denote by $\operatorname{tr}_{*}(g)$ the generating function of traces

$$
\sum_{k, \ell} \operatorname{tr}\left(\left.\tilde{g}\right|_{\mathrm{Sym}^{k}(V) \otimes \Lambda^{\ell} V}\right) T^{k} v^{\ell} .
$$


If $\operatorname{dim}(V)=1$ and $g$ is the multiplication by $a$, then

$$
\operatorname{tr}_{*}(g)=\sum_{k=0}^{\infty} \sum_{\ell=0}^{1} a^{k+\ell} T^{k} v^{\ell}=\frac{1+a v}{1-a T} .
$$

For an automorphism $g: V \rightarrow V$ which is semisimple ${ }^{5}$ on $V$ by (23) and (25) we have

$$
\operatorname{tr}_{*}(g)=\prod_{i=1}^{\operatorname{dim}(V)} \frac{1+a_{i} v}{1-a_{i} T},
$$

where $a_{i}$ for $i=1, \ldots, \operatorname{dim}(V)$ are the eigenvalues of $g$. Hence

$$
\operatorname{tr}_{*}(g)=\frac{\operatorname{det}(1+v g)}{\operatorname{det}(1-T g)} .
$$

To compute the extended Molien series we use the well-known formula

$$
\operatorname{dim}\left(V^{G}\right)=\frac{1}{|G|} \sum_{g \in G} \operatorname{tr}(g),
$$

which applied to every summand $\operatorname{Sym}^{k}(V) \otimes \Lambda^{\ell} V$ instead of $V$ gives us

$$
\operatorname{Mol}(v, T)=\frac{1}{|G|} \sum_{g \in G} t r_{*}(g) .
$$

We obtain

$$
\operatorname{Mol}(v, T)=\frac{1}{|G|} \sum_{g \in G} \frac{\operatorname{det}(1+v g)}{\operatorname{det}(1-T g)},
$$

where $a_{i}(g)$ for $i=1, \ldots, \operatorname{dim}(V)$ are the eigenvalues of $g \in G$ acting on $V$. If the action of $G$ is replaced by the dual action on $V^{*}$ then the Molien series remains unchanged, since the eigenvalues of $g$ are the same as the eigenvalues of $\left(g^{*}\right)^{-1}$.

\section{References}

[AB84] M. F. Atiyah, R. Bott, The moment map and equivariant cohomology, Topology 23 (1984), 1-28.

[Alu13] P. Aluffi, Grothendieck classes and Chern classes of hyperplane arrangements, Int. Math. Res. Not. IMRN 2013 (2013), no. 8, 1873-1900.

[AM09] P. Aluffi, L. C. Mihalcea, Chern classes of Schubert cells and varieties, J. Algebraic Geom. 18 (2009), no. 1, 63-100.

[AW14] M. Andreatta, J. A. Wiśniewski, 4-dimensional symplectic contractions, Geom. Dedicata 168 (2014), 311-337.

[Bat99] V. V. Batyrev. Non-Archimedean integrals and stringy Euler numbers of logterminal pairs, J. Eur. Math. Soc. (JEMS) 1 (1999), no. 1, 5-33.

\footnotetext{
${ }^{5}$ Semisimplicity can be dropped.
} 
[Bau82] P. Baum, Fixed point formula for singular varieties, in: Current Trends in Algebraic Topology, Part 2 (London, Ont., 1981), CMS Conf. Proc., Vol. 2, Amer. Math. Soc., Providence, RI, 1982, pp. pages 3-22.

[BC88] P. Baum, A. Connes, Chern character for discrete groups, in: A fête of Topology, Academic Press, Boston, MA, 1988, pp. 163-232.

[BFQ79] P. Baum, W. Fulton, G. Quart, Lefschetz-Riemann-Roch for singular varieties, Acta Math. 143 (1979), no. 3-4, 193-211.

[BKR01] T. Bridgeland, A. King, M. Reid, The McKay correspondence as an equivalence of derived categories, J. Amer. Math. Soc. 14 (2001), no. 3, 535-554 (electronic).

[BL00] L. A. Borisov, A. Libgober, Elliptic genera of toric varieties and applications to mirror symmetry, Invent. Math. 140 (2000), no. 2, 453-485.

[BL03] L. Borisov, A. Libgober, Elliptic genera of singular varieties, Duke Math. J. 116 (2002), no. 2, 319-351.

[BL05] L. Borisov, A. Libgober, McKay correspondence for elliptic genera, Ann. of Math. (2) 161 (2005), no. 3, 1521-1569.

[BS13] G. Bellamy, T. Schedler, On the (non) existence of symplectic resolutions for imprimitive symplectic reflection groups, ArXiv e-prints, September 2013.

[BSY10] J.-P. Brasselet, J. Schürmann, S. Yokura, Hirzebruch classes and motivic Chern classes for singular spaces, J. Topol. Anal. 2 (2010), no. 1, 1-55.

[BV82] N. Berline, M. Vergne. Classes caractéristiques équivariantes. Formule de localisation en cohomologie équivariante, C. R. Acad. Sci. Paris Sér. I Math. 295 (1982), no. 9, 539-541.

[BV97] M. Brion, M. Vergne. An equivariant Riemann-Roch theorem for complete, simplicial toric varieties, J. Reine Angew. Math. 482 (1997), 67-92.

[CDGZ04] A. Campillo, F. Delgado, S. M. Gusein-Zade. Poincaré series of a rational surface singularity, Invent. Math. 155 (2004), no. 1, 41-53.

[Cha85] K. Chandrasekharan, Elliptic Functions, Grundlehren der Mathematischen Wissenschaften, Vol. 281, Springer-Verlag, Berlin, 1985.

[CMSS12] S. E. Cappell, L. G. Maxim, J. Schürmann, J. L. Shaneson, Equivariant characteristic classes of singular complex algebraic varieties, Comm. Pure Appl. Math. 65 (2012), no. 12, 1722-1769.

[Coh80] A. M. Cohen, Finite quaternionic reflection groups, J. Algebra 64 (1980), no. 2, 293-324.

[DBW14] M. Donten-Bury, J. A. Wiśniewski, On 81 symplectic resolutions of a 4-dimensional quotient by a group of order 32, arXiv:1409.4204 (2014).

[Don69] P. Donovan, The Lefschetz-Riemann-Roch formula, Bull. Soc. Math. France 97 (1969), 257-273.

[Ful98] W. Fulton, Intersection Theory, 2nd ed., Ergebnisse der Mathematik und ihrer Grenzgebiete. 3. Folge. A Series of Modern Surveys in Mathematics, Vol. 2 Springer-Verlag, Berlin, 1998.

[Hir56] F. Hirzebruch, Neue Topologische Methoden in der Algebraischen Geometrie, Ergebnisse der Mathematik und ihrer Grenzgebiete (N.F.), Heft 9. SpringerVerlag, Berlin, 1956. 
[Huh16] J. Huh, Positivity of Chern classes of Schubert cells and varieties, J. Algebraic Geom. 25 (2016), no. 1, 177-199.

[Huy05] D. Huybrechts, Complex Geometry, An introduction, Universitext. SpringerVerlag, Berlin, 2005.

[IR96] Y. Ito, M. Reid, The McKay correspondence for finite subgroups of $\operatorname{SL}(3, \mathbf{C})$, in: Higher-dimensional Complex Varieties (Trento, 1994), de Gruyter, Berlin, 1996., pp. 221-240.

[Kal02] D. Kaledin, McKay correspondence for symplectic quotient singularities, Invent. Math. 148 (2002), no 1, 151-175.

[Lib15] A. Libgober, Elliptic genus of phases of $N=2$ theories, Comm. Math. Phys. 340 (2015), no. 3, 939-958.

[LS12] M. Lehn, C. Sorger, A symplectic resolution for the binary tetrahedral group, in: Geometric Methods in Representation Theory. II, Sémin. Congr., Vol. 24, Soc. Math. France, Paris, 2012, pp. 429-435.

[McK80] J. McKay, Graphs, singularities, and finite groups, in: The Santa Cruz Conference on Finite Groups (Univ. California, Santa Cruz, Calif., 1979), Proc. Sympos. Pure Math., Vol. 37, Amer. Math. Soc., Providence, RI, 1980, pp. 183-186.

[MW15] M. Mikosz, A. Weber, Equivariant Hirzebruch class for quadratic cones via degenerations, J. Singul. 12 (2015), 131-140.

[Ohm06] T. Ohmoto, Equivariant Chern classes of singular algebraic varieties with group actions, Math. Proc. Cambridge Philos. Soc. 140 (2006), no. 1, 115134 .

[PT07] A. Pedroza, L. W. Tu, On the localization formula in equivariant cohomology, Topology Appl. 154 (2007), no. 7, 1493-1501.

[Rei97] M. Reid, McKay correspondence, arXiv:alg-geom/9702016 (1997).

[Sai00] M. Saito, Mixed Hodge complexes on algebraic varieties, Math. Ann. 316 (2000), no. 2, 283-331.

[Ver00] M. Verbitsky, Holomorphic symplectic geometry and orbifold singularities, Asian J. Math. 4 (2000), no. 3, 553-563.

[Vey03] W. Veys, Stringy zeta functions for $\mathbb{Q}$-Gorenstein varieties, Duke Math. J. 120 (2003), no. 3, 469-514.

[Wae08a] R. Waelder, Equivariant elliptic genera, Pacific J. Math. 235 (2008), no. 2, $345-377$.

[Wae08b] R. Waelder, Equivariant elliptic genera and local McKay correspondences, Asian J. Math. 12 (2008), no. 2, 251-284.

[Web12] A. Weber, Equivariant Chern classes and localization theorem, J. Singul. 5 (2012), 153-176.

[Web16a] A. Weber, Equivariant Hirzebruch class for singular varieties, Selecta Math. (N.S.) 22 (2016), no. 3, 1413-1454.

[Web16b] A. Weber, Hirzebruch class and Biatynicki-Birula decomposition, Transform. Groups 22 (2017), no. 2, 537-557. 
Open Access This article is distributed under the terms of the Creative Commons Attribution 4.0 International License (http://creativecommons.org/licenses/by/4.0/), which permits unrestricted use, distribution, and reproduction in any medium, provided you give appropriate credit to the original author(s) and the source, provide a link to the Creative Commons license, and indicate if changes were made. 